\title{
UV irradiated hydrogenated amorphous carbon (HAC) materials as a carrier candidate of the interstellar UV bump at $217.5 \mathrm{~nm}$
}

\author{
K. A. K. Gadallah ${ }^{1,2}$, H. Mutschke ${ }^{1}$, and C. Jäger ${ }^{3}$
}

\author{
${ }^{1}$ Astrophysical Institute and University Observatory, Friedrich Schiller University, Schillergässchen 2/3, 07743 Jena, Germany \\ e-mail: Kamel.Gadallah@uni-jena.de \\ 2 Astronomy Dpt., Faculty of Science, Al-Azhar University, Nasr City, PO Box 11884 Cairo, Egypt \\ 3 Institute of Solid State Physics, Friedrich Schiller University of Jena, Helmholtzweg 3, 07743 Jena, Germany
}

Received 6 August 2010 / Accepted 7 January 2011

\begin{abstract}
Context. Hydrogenated amorphous carbon (HAC) materials have been considered as a laboratory analog of cosmic carbonaceous nanoparticles in the interstellar medium (ISM). In the diffuse ISM, UV radiation can modify the electronic and atomic structure of HAC materials.

Aims. Studying structural and optical properties of HAC materials in correlation with UV processing is very important to understand more clearly the effect of the UV radiation on carbonaceous dust grains in the diffuse ISM. This scenario can explain some astronomical spectral features such as the interstellar UV bump at $4.6 \mu \mathrm{m}^{-1}$.

Methods. Laser ablation has been used to produce nano-sized HAC materials which are subsequently irradiated by strong UV doses in a high vacuum. Transmission electron microscope images and spectroscopic analyses show the evolution of the internal structure of the material with the UV irradiation.

Results. It is found that hydrogen content and the $\mathrm{sp}^{3} / \mathrm{sp}^{2}$ hybridization ratio decrease with the UV irradiation. The graphene layers become longer in processed materials. Also, graphitic fibers are observed in modified materials. The variation in the internal structure leads to dramatic changes in the spectral properties in the FUV-VIS range. The UV irradiation of HAC materials, coresponding to $21-33 \%$ of the average dose of the UV radiation in diffuse ISM, has produced a new band centered at $4.6 \mu \mathrm{m}^{-1}(217.5 \mathrm{~nm})$.

Conclusions. Consequently, these results confirm for the first time the suggestion by Mennella et al. (1996) that irradiated HAC materials might be considered the carrier of the interstellar UV bump at $4.6 \mu \mathrm{m}^{-1}$. However, so far the amount of carbon needed to produce the interstellar $4.6 \mu \mathrm{m}^{-1}$ band is higher than that available for interstellar carbon dust grains. So the ideal structure of irradiated HAC materials that would produce a band of sufficient strength is not yet clear for the interstellar dust.
\end{abstract}

Key words. dust, extinction - ISM: lines and bands - ultraviolet: ISM - methods: laboratory

\section{Introduction}

In the diffuse interstellar medium (ISM), carbonaceous grains are extremely important components of the dust (Greenberg \& Li 1999; Ehrenfreund \& Charnley 2000). It is assumed that these are carbonaceous structures, with a mixture of hybridizations $\left(\mathrm{sp}^{3}, \mathrm{sp}^{2}\right.$ and $\left.\mathrm{sp}^{1}\right)$ of carbon atoms, such as hydrogenated amorphous carbon (HAC) materials which contain a fraction of hydrogen. Nowadays, HAC materials have been considered as a realistic model for interstellar carbon dust (e.g., Jones et al. 1990; Dartois et al. 2004, 2007; Dartois \& Muñoz-Caro 2007; Pino et al. 2008). In astronomical observations in MIR range, the $\mathrm{C}-\mathrm{H}$ stretching vibration band at $3.4 \mu \mathrm{m}$ and the $\mathrm{C}-\mathrm{H}$ deformation bands at 6.85 and $7.25 \mu \mathrm{m}$ are evidences of the existence of HAC materials in the diffuse ISM (Chiar et al. 2000; Pendleton \& Allamandola 2002).

Once carbonaceous grains are injected into the diffuse cloud, they are bathed in an UV radiation field (Ogmen \& Duley 1988; Rietmeijer 1988). HAC materials can be transformed from amorphous diamond-like $\left(\mathrm{sp}^{3}\right)$ to much more graphitic $\left(\mathrm{sp}^{2}\right)$ carbon. The energy of UV photons is high enough to break C$\mathrm{H}$ bonds because the bond energy of the $\mathrm{C}-\mathrm{H}$ bond is about $5 \mathrm{eV}$ $(\sim 250 \mathrm{~nm})$, while UV flux photons have energies between 5 and $13 \mathrm{eV}$ (Buch 1989). Due to this destruction of the chemical network of HAC material, the librated energy would heat up the material enough to remove $\mathrm{H}$ atoms before the recombination of some $\mathrm{H}$ atoms (depending on the degree of the hydrogenation, Mennella et al. 2002) proceeds again. Consequently, the structure of HAC materials is profoundly modified by the interstellar UV radiation flux. This flux is given by $F \cong F_{0} \exp \left(-2 A_{\mathrm{V}}\right)$, where $A_{\mathrm{V}}$ is the visual extinction and $F_{0}=9.6 \times 10^{8} \mathrm{eV} \mathrm{cm}^{-2} \mathrm{~s}^{-1}$ is the unattenuated flux in the range 91-180 nm (Habing 1968; Mathis et al. 1983). Under the conditions of diffuse interstellar clouds $\left(A_{\mathrm{V}}=0.6\right), F$ is about $3 \times 10^{8} \mathrm{eV} \mathrm{cm}^{-2} \mathrm{~s}^{-1}$. Hence, the total dose (D) of UV photons deposited on amorphous carbon grains is $3 \times 10^{23} \mathrm{eV} \mathrm{cm}^{-2}$ during the lifetime of the diffuse cloud $\left(3 \times 10^{7} \mathrm{yr}\right)$ (Jenniskens 1993). For diffuse clouds the UV irradiation time-scale for photofission of C-H bonds is $1.2 \times 10^{6} \mathrm{yr}$ (for more details see Sorrell 1990). It is short compared to the grain processing time corresponding to the cloud lifetime.

The wavelength-dependence of the interstellar extinction curve provides important diagnostic informations about the physical and chemical properties of interstellar dust grains and always serves as a first test for grain models. The structure of UV extinction curves including the $4.6 \mu \mathrm{m}^{-1}$ (217.5 nm) bump has been explained by Fitzpatrick \& Massa (1990); Fitzpatrick (1999), and the most recent studies have provided a general survey of galactic extinction covering the near-IR (NIR) through UV spectral regions (Fitzpatrick \& Massa 2005, 2007). The remarkable aspect of the extinction curve is the broad UV bump 
centered at $4.6 \mu \mathrm{m}^{-1}$. This peak is still the most controversial feature, concerning its origin, in the diffuse interstellar medium. Since the first observation of the $4.6 \mu \mathrm{m}^{-1}$ bump (Stecher 1965) until the modern astronomical data (Whittet 2004; Sofia et al. 2005; Fitzpatrick \& Massa 2007), observations have shown that the peak postion of this bump is very stable at $4.6 \mu \mathrm{m}^{-1}$, while its width varies from 36 to $77 \mathrm{~nm}$. The attribution of this band has been a long-standing problem. Here the tricky point is to explain the extreme constancy of the peak position associated to band width variations.

Regarding the carrier of the interstellar UV bump at $4.6 \mu \mathrm{m}^{-1}$, it was thought that small crystalline graphite particles are the carrier of this bump (e.g., Draine \& Lee 1984). The recent laboratory analogs (by e.g., Colangeli et al. 1990, 1997; Schnaiter et al. 1998; Furton et al. 1999; Mennella et al. 1999; Jäger et al. 1999, 2008) and the theoretical studies (Hecht 1986; Sorrell 1990; Duley \& Seahra 1998), have developed a different approach that the carrier of this band is a hydrogenated carbonaceous solid including $\mathrm{CH}, \mathrm{CH}_{2}, \mathrm{CH}_{3}$ chemical groups in connection with a graphitic backbone. The analyses by these authors (and others) on submicron-sized amorphous carbon grains have considered an interstellar amorphous carbon whose properties are able to explain many astronomical features. Carbonaceous materials have been produced by using different techniques and having a different degree of structural order, hydrogen content, and grain size. Studies by Mennella et al. (1996, 1997, 1998); Schnaiter et al. (1998); Wada et al. (1999); Chhowalla et al. (2003) have generally shown that HAC materials, through their electronic structure, could play an important role as carrier of the UV bump. The UV processing is proposed to play an important role in the electronic structure of this material. Mennella et al. (1996) attributed this band to the $\pi-\pi^{*}$ electronic transitions of the delocalized electrons in $\mathrm{sp}^{2}$ hybridized carbon bonds in carbon clusters. They found a band at $215 \mathrm{~nm}$ $\left(4.65 \mu \mathrm{m}^{-1}\right)$ after exposing the HAC materials to UV irradiation of $(1-4) \times 10^{22} \mathrm{eV} \mathrm{cm}^{2}$ which is corresponding to $(3-13) \%$ of the average value of the interstellar radiation dose.

For understanding better this scenario which simulates the interaction of the HAC materials with UV radiation in the diffuse interstellar medium, it is important to explain (by microscopic and spectroscopic methods) the effect of UV radiation on the structure of HAC materials. In this work, we study the modification in the structure and optical properties (in a wider range from the FUV to NIR and in the MIR range) before and after exposing HAC materials to higher UV irradiation doses. The production and the UV processing of the samples are shown in Sect. 2. The results and discussion are given in Sect. 3, which explains how the optical properties are related to the modifications in the structure of HAC materials due to the UV irradiation. These modifications include changes of the length of the graphene layers $\left(L_{\mathrm{a}}\right)$, grain sizes, hydrogen content and $\mathrm{sp}^{3} / \mathrm{sp}^{2}$ hybridization ratio as well as of the gap energy $E_{\mathrm{g}}$. In Sect. 4 , the spectral characteristics of irradiated HAC materials are compared with the interstellar UV bump at $4.6 \mu \mathrm{m}^{-1}$. The total amount of carbon which is needed to match band strengths in the ISM is taken into account. Finally, the conclusion is given in Sect. 5 .

\section{Experiments}

\subsection{Sample production}

In order to produce HAC materials, the laser ablation of a solid material has been used. We use a Nd:YAG laser which gives very short and intense pulses with a wavelength of $532 \mathrm{~nm}$ (second harmonic) at a pulse rate of $10 \mathrm{~Hz}$. It is used to heat a graphite surface and to vaporize a small quantity of graphite as an intense plasma taking a plume-like shape depending on the ambient gas pressure. Collisions between the evaporated carbon atoms and also between clusters and of all of these with the quenching gas $\left(\mathrm{He} / \mathrm{H}_{2}\right)$ in a supersonic expansion of the hot plasma cause the condensation of carbon particles. The laser ablation method is further characterized by the parameter laser power density $\left(F_{1}\right)$ which is again controlled by the area of the laser beam focus on the target. The duration of the laser pulses, $t$, is $10 \mathrm{~ns}$ with pulse energies $E_{\text {pulse }}$ ranging from 60 to $220 \mathrm{~mJ}$ measured with a powerlite meter (Continuum C5100) ranging from 1 to 2.2 Watt. The laser power density has been determined for each laser power from the following equations,

$F_{1}=\frac{E_{\text {pulse }}}{t \pi r^{2}}$

where $r$ is the radius of the focused area of the laser beam. The value $F_{1}$ is $2 \times 10^{8} \mathrm{~W} \mathrm{~cm}^{-2}$ in the case of low-focused laser beam (with $r \approx 1 \mathrm{~mm}$ ). This value results in a plasma with a vibrational temperature of approximately $4000 \mathrm{~K}$ in the condensation zone (Jäger et al. 2008).

HAC samples are produced by pulsed-laser ablation of a rotating graphite target in a quenching gas atomsphere which consists of a mixture of $\mathrm{He}$ and $\mathrm{H}_{2}$ gas at a pressure of 4.5 mbar. Both gases are continuosly supplied because there is a loss through the nozzle to the expansion chamber which is continuously pumped. The gas flows are controlled by a "multi gas controller 647B (MKS)". In order to avoid strong structural processing and agglomeration of the original condensed grains, particles are directed from the condensation zone through the nozzle and skimmer to form a freely propagating particle beam throughout the low-pressure atmosphere in the expansion and analysing chambers (see Fig. 1 in Jäger et al. 2008). Then they are deposited in a separated chamber (substrate chamber) at a pressure of $(5 \pm 3) \times 10^{-6}$ Torr. The amount of deposited carbon materials i.e. the areal mass density (the mass per unit area, $\rho_{\mathrm{a}}$ ) on the substrate $\left(\mathrm{SiO}_{2}\right.$ and $\mathrm{CaF}_{2}$, or $\mathrm{KBr}$ substrates) has been measured by means of a quartz microbalance which is sharing the cross section of the particle beam with the substrate. The deposits on both the microbalance and the substrate are rather uniform. The areal mass densities are corrected to the real coverage area on the microbalance, because the value given on the microbalance display is based on the assumption of full coverage.

HAC samples have been produced under physical conditions shown in Table 1. The gaseous atmosphere in the deposition chamber is $\mathrm{He}$ and $\mathrm{H}_{2}$ with a ratio of $\mathrm{He} / \mathrm{H}_{2}$ ranging from 1.7 to 12.5 . In order to increase the hydrogen content of the produced HAC materials, we increased the laser power density by approximately two orders of magnitude in the case of a highfocused laser beam (with $r \approx 0.15 \mathrm{~mm}$ ). Accordingly, this higher laser power density rises the vibrational temperature of the laserinduced plasma to a value larger than $4000 \mathrm{~K}$ (Iida \& Yeung 1994). With the gas atmosphere used at low laser power density we do not get any deposition, therefore we changed the $\mathrm{He} / \mathrm{H}_{2}$ ratio to higher values. More dissociative carbon atoms and even small carbon molecules are formed due to the increase of the laser power density (Iida \& Yeung 1994). This is probably the reason why these conditions result in a high degree of hydrogenation (see Sect. 3.2.1) even at a lower hydrogen fraction in the atmosphere. We note that Jäger et al. (2008) found a relatively high hydrogenation $(\mathrm{H} / \mathrm{C}=0.5)$ also at quite low laser power densities, which may point to different reaction mechanisms at these conditions. Because the high laser power density results in a more rapid expansion of the plasma, the higher 
helium content is needed to confine the plasma by collisions to allow for an efficient condensation of solid HAC particles. Alternatively, the lower hydrogen content may result in a reduction of the formation of volatile species.

\subsection{UV processing}

For UV irradiation, we use a deuterium lamp (L2D2 lamp, type L7292, from HAMAMATSU). It is a discharge light source that utilizes stable arc discharge in deuterium gas $\left(\mathrm{D}_{2}\right)$. According to the spectrum provided by the producer of the lamp, we can approximately calculate the radiation intensity or the radiant power, $P_{\mathrm{ph}}$, of the lamp by integrating the area under this curve. The radiation is emitted into a solid angle of 10 degrees (from the technical specification) allowing to use the entire flux. The molecular emission of this curve is dominant at the wavelength $\lambda=160 \mathrm{~nm}$. Its spectral distribution is $0.5 \mathrm{~mW}$ at $\lambda<155 \mathrm{~nm}$, and $0.4 \mathrm{~mW}$ in the range $155 \mathrm{~nm}<\lambda<165 \mathrm{~nm}$, and $0.4 \mathrm{~mW}$ at $165 \mathrm{~nm}<\lambda<230 \mathrm{~nm}$. The total value of $P_{\mathrm{ph}}$ is $1.3 \mathrm{~mW}$ over the whole band of the VUV range. We expect that this value may have an uncertainty of about $\pm 20 \%$.

This value agrees quite well with the value measured by using a platinum photocathode at a voltage of $15 \mathrm{~V}$, in high vacuum $\left(\approx 10^{-6}\right.$ mbar). The photocurrent of the irradiated photoelement was $3.0 \mu \mathrm{A}$. The number of electrons, $n_{\mathrm{e}}$, emitted from the photocathode is therefore:

$n_{\mathrm{e}}=\frac{3.0 \times 10^{-6}(\mathrm{~A})}{1.6 \times 10^{-19}(\mathrm{As})}=1.8 \times 10^{13}\left(\mathrm{~s}^{-1}\right)$.

By using a platinum photocathode, only photons with energies above $5.66 \mathrm{eV}$ ( $\lambda$ lower than $220 \mathrm{~nm}$ ) can be measured. The photoelectric efficiency of the platinum slice at $120 \mathrm{~nm}$ (Cairns $\&$ Samson 1966) is approximately 0.02. By extrapolating to $\lambda=160 \mathrm{~nm}$, this value is expected to be considerably lower. If we assume an average value of 0.015 in the spectral range of the deuterium lamp flux, the number of photons $n_{\mathrm{ph}}$ is $1.2 \times 10^{15}$ photons $\mathrm{s}^{-1}$. The power of the total flux of photons $\left(P_{\mathrm{ph}}\right)$, assuming an average photon energy $\left(E_{\mathrm{ph}}\right)$ of $7.75 \mathrm{eV}(\lambda=160 \mathrm{~nm})$, at the sample position is

$P_{\mathrm{ph}}=1.6 \times 10^{-19}\left(\frac{\mathrm{Ws}}{\mathrm{eV}}\right) \times n_{\mathrm{ph}} \times E_{\mathrm{ph}}=1.4 \mathrm{~mW}$.

The uncertainty of the latter value should be much larger, so we prefer to use the first value estimated from the producers data for calculating the UV doses. The long term stability of the deuterium lamp had been checked by measuring the platinum photocurrent again after the long periods of irradiation (about $500 \mathrm{~h}$ ). We found that the radiant power of the lamp did not change during the irradiation time which is approximately $25 \%$ of the life time $(2000 \mathrm{~h})$ of the lamp.

Selected samples have been irradiated at room temperature in a chamber with high vacuum $\left(\approx 3 \times 10^{-6} \mathrm{mbar}\right)$. The whole radiation coming from the lamp is collected by a concave mirror ( $5 \mathrm{~cm}$ in diameter) located $10 \mathrm{~cm}$ from the lamp. This mirror focuses the UV beam on a small area $(1.5 \mathrm{~mm}$ in diameter) on the sample. The reflectivity of this mirror is assumed to reduce the UV flux by $20 \%$ resulting in UV flux of $3.68 \times 10^{17} \mathrm{eV} \mathrm{s}^{-1} \mathrm{~cm}^{-2}$. Table 1 shows the UV doses that fell on each sample during the UV irradiation time per irradiated area. This high UV flux has some (quantitatively unknown) local heating effect on the structure of HAC materials. It is certainly much smaller comparing to the effect of annealing of the same kind of materials. The later effect will be discussed in another publication which is in preparation.

\subsection{Transmission electron microscopy (TEM)}

In addition to the spectroscopy, other kinds of information about the chemical composition and the structural arrangement of HAC nanoparticles can be obtained by electron microscopy. The internal structure of carbonaceous material can be described by using the TEM in its high resolution mode (HRTEM). HRTEM images have been taken with a JEOL JEM-3010 microscope equipped with $\mathrm{a} \mathrm{LaB}_{6}$ cathode working at an acceleration voltage of $300 \mathrm{kV}$. For this purpose, the condensed particles were either directly deposited on the TEM grids in the generated particle beam or they were removed from the substrate and a dispersion of the grains in chloroform was dropped on the grids. In the high-resolution mode, the length and structure (bent or plane) of the graphene layers are studied.

Studies (Jäger et al. 1998, 2008; Mikhailov et al. 2001), done by HRTEM at high magnification allowing to resolve more details of physical structure, have shown that carbonaceous nanoparticles consist of small spherical primary particles having sizes between a few and tens of nanometers. Frequently, they are incorporated in branched aggregates. They are a mixture of amorphous elemental carbon and some amount of highmolecular hydrocarbons.

\subsection{Optical transmission measurements}

The attenuation of transmitted light by a medium consisting of particles is also called extinction, which can be expressed by the sum of the extinction cross sections $\left(C_{\text {ext }}\right)$ of the particles. The extinction cross section contains the contributions of both the absorption of radiation by the materials and the scattering by the sample's inhomogeneity, $C_{\text {ext }}=C_{\text {abs }}+C_{\text {sca }}$. The terms $C_{\mathrm{abs}}$ and $C_{\mathrm{sca}}$ are the absorption and scattering cross sections, respectively. When the scattering contribution is negligible as in our original HAC materials because the particles are very small, the absorption is the dominant mode of the extinction. The extinction cross section per unit particle mass $\left(m_{\mathrm{p}}\right)$ is equal to the mass absorption coefficient $(\kappa)$ expressed in $\mathrm{g}^{-1} \mathrm{~cm}^{2}$. If the sample would consist of isolated particles, $\kappa$ could be directly derived for these particles from the measured transmission $(T)$ and the areal mass density of the sample as the following

$\frac{C_{\mathrm{ext}}}{m_{\mathrm{p}}}=\kappa=-\ln T \frac{1}{\rho_{\mathrm{a}}}$.

If the particles are agglomerated as in case of our samples this relation can only be considered a first-order approximation. Mennella et al. (1998) had used a nonspherical-particle model (CDE; continuous distribution of ellipsoids) for describing the state of agglomerated particles and correcting for the effect of agglomeration. In case of our irradiated materials, unfortunately this approach is not applicable because of the large particle size. Therefore, we have to rely on the approximate formula, Eq. (4), which does not correct for agglomeration effects. Within this limitation, the extinction cross section per number of carbon atoms can be expressed in terms of $\kappa$ multiplied by the atomic mass of carbon in case if we ignore the mass of the hydrogen content compared to the mass of the carbon amount,

$\frac{C_{\mathrm{ext}}}{N_{\mathrm{C}}} \approx \kappa m_{\mathrm{C}} \approx \kappa\left(12 m_{\mathrm{H}}\right)$

where $m_{\mathrm{H}}$ is the atomic hydrogen mass.

The spectroscopic measurements in the VIS and UV regions have been performed by a commercial spectrophotometer 
Table 1. Physical conditions to produce HAC samples in a gas pressure equal to 4.5 Torr.

\begin{tabular}{|c|c|c|c|c|c|c|c|c|c|c|c|}
\hline \multirow[b]{2}{*}{$\mathrm{S} \#^{1}$} & \multirow[b]{2}{*}{$\begin{array}{c}\rho_{\mathrm{a}} \\
\left(10^{-7} \mathrm{~g} \mathrm{~cm}^{-2}\right) \\
\end{array}$} & \multirow[b]{2}{*}{$\begin{array}{c}\text { Atmosphere } \\
\left(\mathrm{He}: \mathrm{H}_{2}\right)\end{array}$} & \multirow[b]{2}{*}{$\begin{array}{c}F_{1} \\
\left(\mathrm{~W} \mathrm{~cm}^{-2}\right)\end{array}$} & \multicolumn{4}{|c|}{ Before UV irradiation } & \multicolumn{4}{|c|}{ After UV irradiation } \\
\hline & & & & $\begin{array}{c}k_{\mathrm{CH}} \\
\left(\mathrm{cm}^{2} \mathrm{~g}^{-1}\right)\end{array}$ & $\mathrm{H} / \mathrm{C}$ & $\mathrm{sp}^{3} / \mathrm{sp}^{2}$ & $\begin{array}{c}E_{\mathrm{g}} \\
(\mathrm{eV})\end{array}$ & $\begin{array}{c}\text { UV dose } \\
\left(\mathrm{eV} \mathrm{cm}^{-2}\right)\end{array}$ & $\mathrm{H} / \mathrm{C}$ & $\mathrm{sp}^{3} / \mathrm{sp}^{2}$ & $\begin{array}{c}E_{\mathrm{g}} \\
(\mathrm{eV})\end{array}$ \\
\hline S1 & 116.25 & $50: 30$ & $2 \times 10^{8}$ & 45 & 0.60 & 0.40 & 1.1 & $\ldots$. & $\ldots$. & $\ldots$. & $\ldots$. \\
\hline $\mathrm{S} 2$ & 41.85 & $50: 15$ & $2 \times 10^{8}$ & $\ldots$. & $\ldots$. & $\ldots$. & 1.2 & $\ldots$ & $\ldots$. & $\ldots$. & $\ldots$. \\
\hline $\mathrm{S} 3$ & 48.05 & $50: 30$ & $2 \times 10^{8}$ & $\ldots$. & $\ldots$. & $\ldots$. & 1.27 & $5.2 \times 10^{22}$ & $\ldots$. & $\ldots$. & $\ldots$. \\
\hline S3(grid) & $\cdots$ & $50: 30$ & $2 \times 10^{8}$ & $\cdots$ & $\cdots$ & $\cdots$ & $\ldots$ & $\begin{array}{l}3.0 \times 10^{22} \\
1.0 \times 10^{23}\end{array}$ & $\ldots$ & $\cdots$ & $\cdots$ \\
\hline- & $\ldots$ & . - & - - & - & - & $-\cdots$ & $-\cdots$ & $2.9 \times 10^{22}$ & $\begin{array}{l}\cdots \cdot- \\
\cdots \cdots\end{array}$ & $\cdots$ & $\begin{array}{l}\cdots- \\
\cdots\end{array}$ \\
\hline S4 & 58.9 & $50: 30$ & $2 \times 10^{8}$ & 49 & 0.62 & 0.43 & 1.21 & $6.3 \times 10^{22}$ & $\ldots$ & $\ldots$ & $\ldots$ \\
\hline & & & & & & & & $1.0 \times 10^{23}$ & 0.43 & 0.19 & 1.37 \\
\hline S5 & 51.15 & $50: 15$ & $2 \times 10^{8}$ & $\ldots$ & $\ldots$ & $\ldots$ & 1.32 & $\ldots$ & $\ldots$ & $\ldots$ & $\ldots$ \\
\hline S6 & 38.75 & $50: 15$ & $2 \times 10^{8}$ & $\ldots$ & $\ldots$. & $\ldots$ & 1.42 & $\ldots$ & $\ldots$. & $\ldots$. & $\ldots$. \\
\hline S7 & 38.75 & $50: 30$ & $2 \times 10^{8}$ & $\ldots$ & $\ldots$ & $\ldots$ & 1.45 & $1.0 \times 10^{23}$ & $\ldots$ & $\ldots$. & $\ldots$ \\
\hline S8 & 34.1 & $50: 30$ & $2 \times 10^{8}$ & $\ldots$ & $\ldots$ & $\ldots$. & 1.33 & $6.3 \times 10^{22}$ & .... & $\ldots$. & 1.67 \\
\hline S9 & 57.35 & $50: 15$ & $2 \times 10^{8}$ & .... & .... & .... & 1.5 & .... & .... & .... & .... \\
\hline S10 & 46.5 & $50: 30$ & $2 \times 10^{8}$ & .... & .... & $\ldots$. & 1.5 & .... & .... & .... & .... \\
\hline S11 & 89.9 & $50: 30$ & $2 \times 10^{8}$ & 47 & 0.61 & 0.41 & 1.55 & $\ldots$ & $\ldots$ & $\ldots$ & $\ldots$. \\
\hline S14 & 100.75 & $90: 20$ & $2.8 \times 10^{10}$ & 66 & 0.70 & 0.55 & 1.65 & .... & .... & $\ldots$. & $\ldots$. \\
\hline S15 & 124.0 & $150: 12$ & $2.0 \times 10^{10}$ & 92 & 0.78 & 0.70 & 1.7 & .... & .... & .... & .... \\
\hline S16 & 62.0 & $80: 20$ & $2.1 \times 10^{10}$ & 99 & 0.79 & 0.74 & 1.7 & .... & $\ldots$. & $\ldots$. & $\ldots$. \\
\hline S17 & 62.0 & $100: 30$ & $2.1 \times 10^{10}$ & 47 & 0.61 & 0.42 & 1.72 & .... & $\ldots$. & $\ldots$. & $\ldots$. \\
\hline S18 & 100.75 & $90: 20$ & $2.8 \times 10^{10}$ & 85 & 0.76 & 0.67 & 1.8 & $\ldots$. & $\ldots$. & $\ldots$. & $\ldots$. \\
\hline S19 & 100.75 & $90: 20$ & $2.8 \times 10^{10}$ & $\ldots$ & $\ldots$ & $\ldots$. & 1.8 & $\ldots$ & $\ldots$ & $\ldots$ & $\ldots$ \\
\hline S20 & 93.0 & $80: 20$ & $1.4 \times 10^{10}$ & 65 & 0.69 & 0.55 & 1.8 & $\ldots$ & $\ldots$ & $\ldots$ & $\ldots$ \\
\hline S21 & 62.0 & $90: 10$ & $2.0 \times 10^{10}$ & $\ldots$ & $\ldots$ & $\ldots$. & 1.75 & $6.3 \times 10^{22}$ & $\ldots$ & $\ldots$ & 1.68 \\
\hline $\mathrm{S} 22$ & 116.25 & $90: 20$ & $2.8 \times 10^{10}$ & 89 & 0.77 & 0.69 & 1.82 & .... & .... & $\ldots$. & $\ldots$ \\
\hline $\mathrm{S} 23$ & 38.75 & 50:20 & $3.0 \times 10^{10}$ & $\ldots$ & $\ldots$. & $\ldots$. & 2.15 & $1.0 \times 10^{23}$ & $\ldots$ & $\ldots$ & 1.6 \\
\hline $\mathrm{S} 24$ & 186.0 & $90: 20$ & $2.8 \times 10^{10}$ & 113 & 0.82 & 0.80 & $\ldots$ & $2.1 \times 10^{22}$ & 0.70 & 0.56 & .... \\
\hline S26 & 141.05 & 50:20 & $3.1 \times 10^{10}$ & 81 & 0.75 & 0.65 & $\ldots$. & $1.0 \times 10^{23}$ & 0.37 & 0.14 & $\ldots$ \\
\hline
\end{tabular}

Notes. ${ }^{(1)} \mathrm{S} 8$, S23, and $\mathrm{S} 24$ are deposited on a $\mathrm{CaF}_{2}$ substrate, while $\mathrm{S} 26$ is deposited on a $\mathrm{KBr}$ substrate. Other samples except S3(grid) are deposited on a $\mathrm{SiO}_{2}$ substrate. S1, S2, S3, S3(grid), S7, S22, S24 and S26 are used for HRTEM images, and for UV irradiation S1, S3(grid), S4, S7, S8, S21, S23, S24, and S26 are used. In order to follow the evolution of the small rise which appears mostly in the FUV band, S1-S11 and S14-S23 are used.

(Perkin Elmer, UV/VIS/NIR Lambda 19). In the FUV measurements, an additional VUV Spectrophotometer (deuterium $\mathrm{D}_{2-}$ lamp) which is produced by the Laser Zentrum Hannover has been used. The transmission spectra in the MIR have been taken by using FTIR spectrometer Bruker 113v. All spectroscopic measurements have been done in ex situ on spots smaller than the irradiated areas by using beam cross sections of typically $1 \mathrm{~mm}$ in diameter (1.5 mm for FTIR measurements). For adapting the spectrometer beam size, a mask has been used. Finding the irradiated spot for the measurements was easily possible, because the sample color became darker after irradiation.

\section{Results and discussions}

\subsection{Analytical high resolution transmission electron microscopy (HRTEM) of HAC materials}

By using different (low and high) laser power densities (Table 1), slight differences in the structure can be observed in the deposit as shown in Fig. 1. The comparison of the HRTEM images has shown that at low laser power density the material contains more pores as shown in panels $(a),(b)$ and $(c)$. In the panel $d$, the disordered structure produced at higher laser power density, in contrast is very dense. This is probably due to the stronger evaporation and higher condensation rate of carbonaceous particles. The deposited grains shown in panels $(a),(b)$ and $(c)$ are in a smallsize range $(2-4 \mathrm{~nm})$ whereas the grain size of the material shown in the panel $(d)$ is not clear because it is very dense. In panels $(a)$, (b) and $(d)$, the particles are mostly fragments and have chainlike shape. For the material in the panel $(c)$, the agglomerates are mostly closed fullerene-like carbon materials. It is probably because of the low fraction of the incorporated hydrogen. A typical appearance of the disordered strongly bent graphene layers and small fullerene-like carbon grains is observed in all materials. As shown in Table 2, which summarizes the average length of the graphene layers $\left(L_{\mathrm{a}}\right)$ of original and processed HAC materials, $L_{\mathrm{a}}$ of the original materials is roughly between $0.42 \mathrm{~nm}$ (at using low laser power density) and $0.72 \mathrm{~nm}$ (at using high laser power 
K. A. K. Gadallah et al.: UV irradiated hydrogenated amorphous carbon (HAC) materials
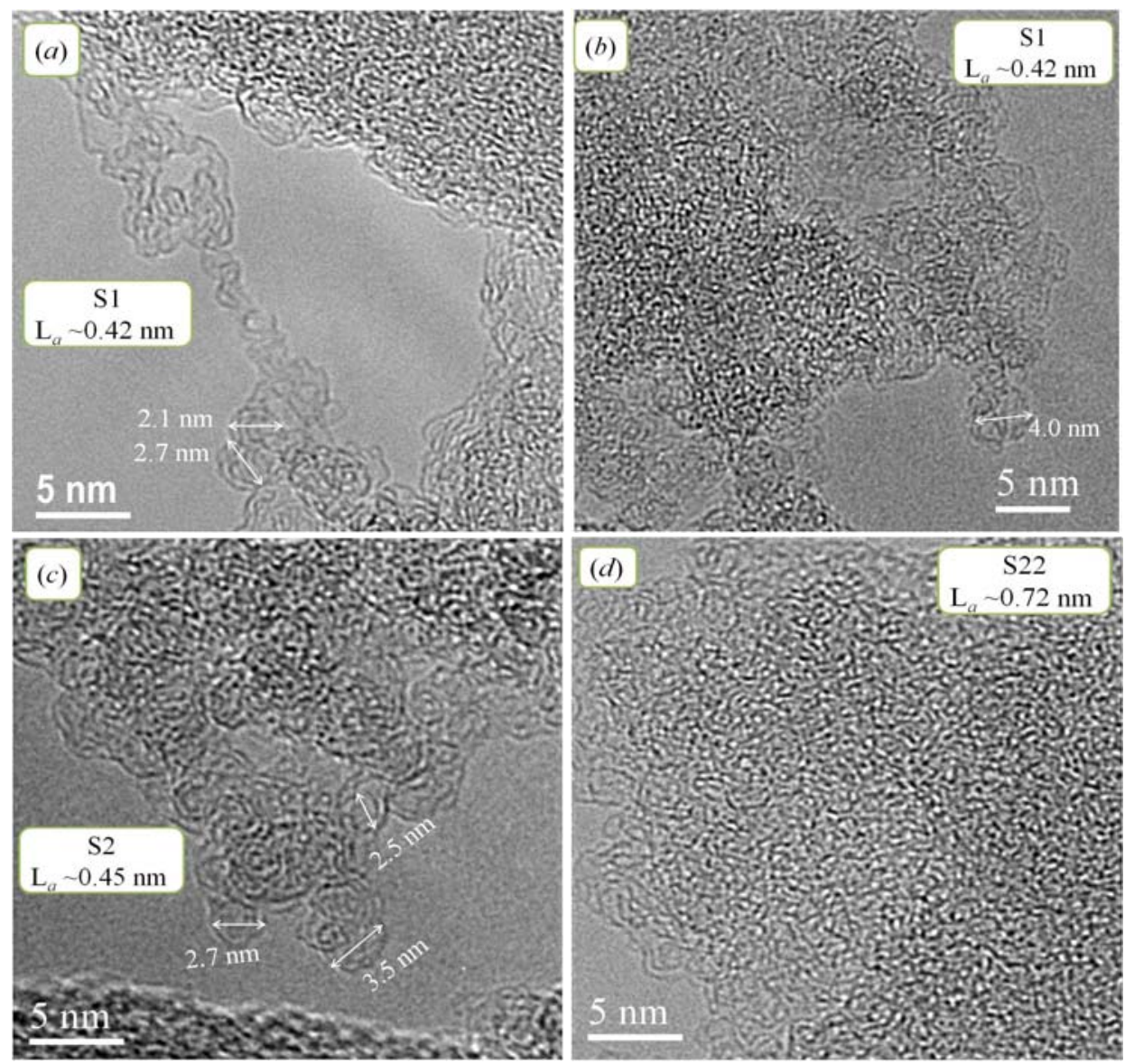

Fig. 1. HRTEM images of HAC materials produced at low laser power density (S1 and S2) and at high laser power density (S22).
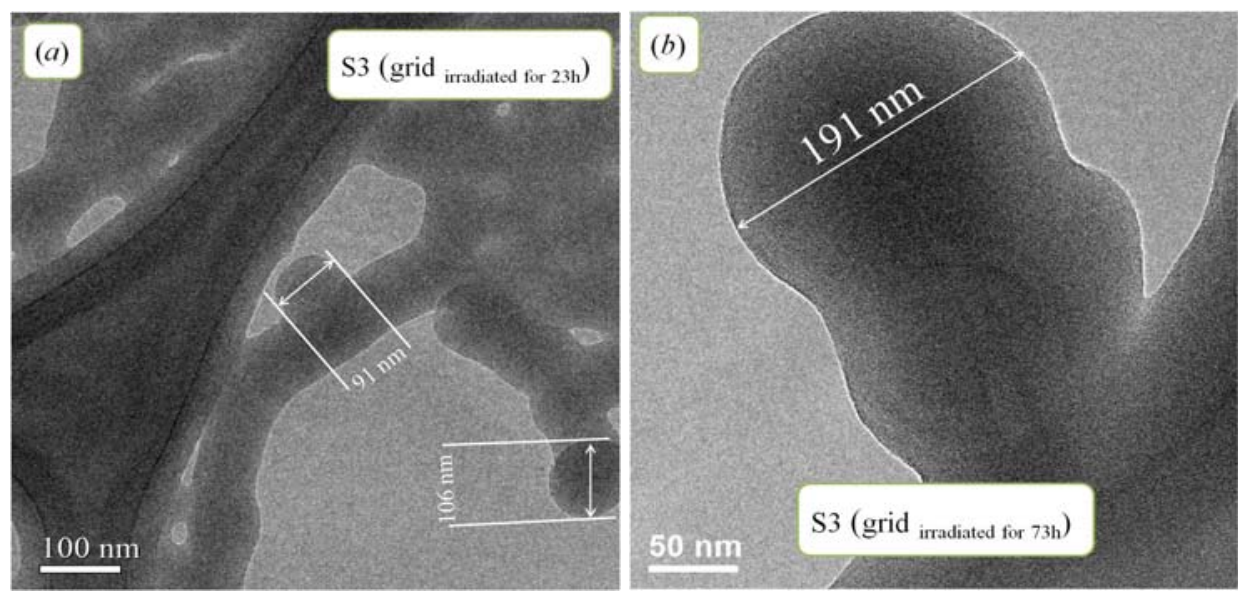

Fig. 2. Low resolution images showing the morphology of HAC particles irradiated on the grid for $23 \mathrm{~h}(a)$ and $72 \mathrm{~h}(b)$.

density). This is consistent with what is observed by Jäger et al. (2008).

The amorphous structure of the carbonaceous grains can be modified after UV irradiation resulting in more graphitic structures. The degree of the graphitization depends on the UV dose which is incident on the HAC material during the irradiation time. For a HAC material S3(grid) deposited directly on the grid in the analyzing chamber, it has been exposed to UV doses which are $3.0 \times 10^{22} \mathrm{eV} \mathrm{cm}^{-2}$ during 23 hours and $1.0 \times 10^{23} \mathrm{eV} \mathrm{cm}^{-2}$ during 72 h. In Fig. 3, HRTEM images show the development of the grain size with the UV irradiation. According to the influence of these doses, the grain size increases with the UV irradiation to be 91-106 nm (panel $(a)$ ) and $191 \mathrm{~nm}$ (panel (b)), respectively. Figure 3 shows the change in the internal structure of the particles with irradiation causing mainly increasing graphitization. This is described through the variation of $L_{\mathrm{a}}$ with the UV irradiation. In the panel (a), a relatively even modification of the disordered layers has been observed at lower UV dose $\left(3.0 \times 10^{22} \mathrm{eV} \mathrm{cm}^{-2}\right)$. The value of $L_{\mathrm{a}}$ becomes $1.72 \mathrm{~nm}$ and a few graphite fibers can be observed. With higher UV irradiation 

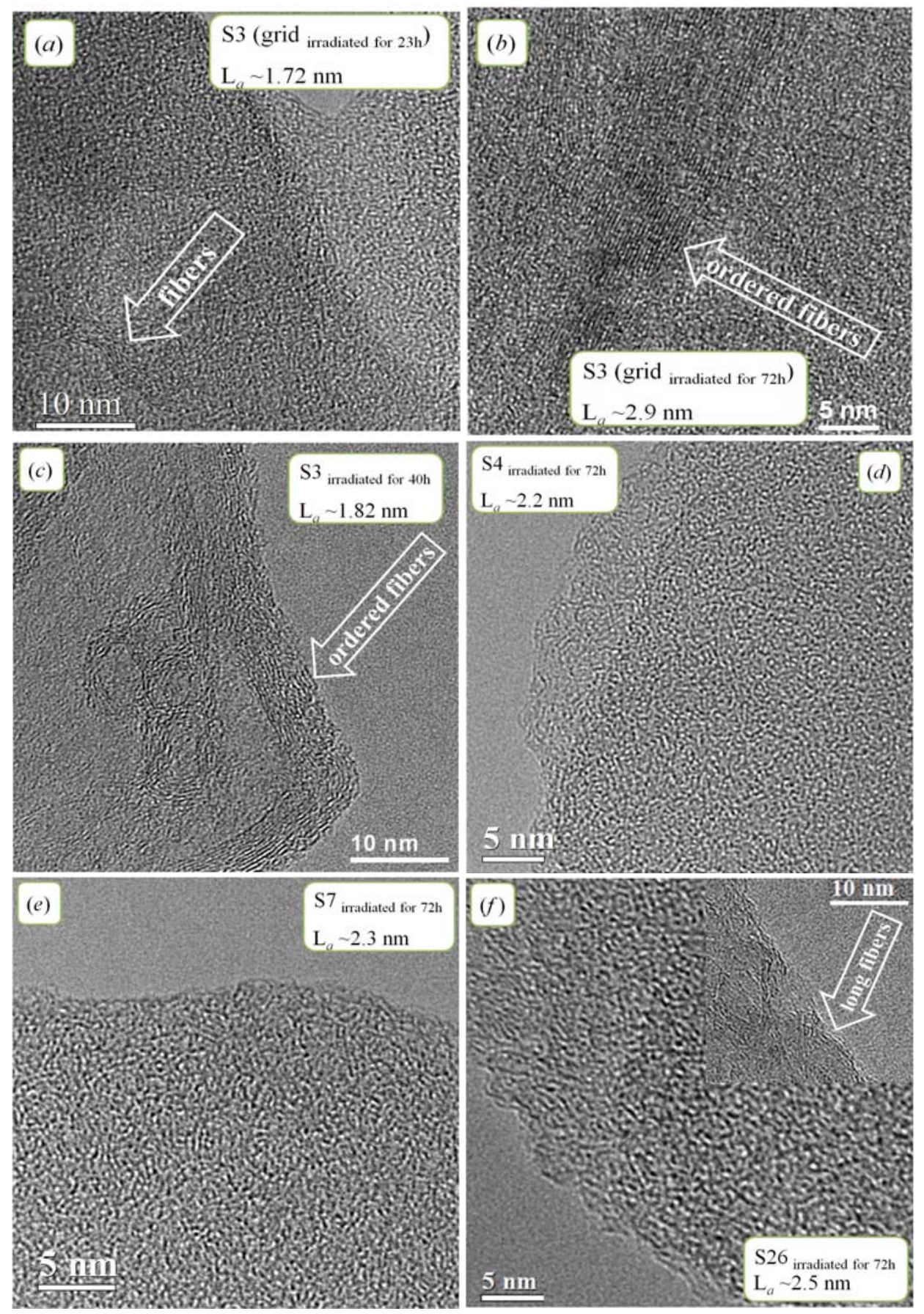

Fig. 3. HRTEM images of irradiated HAC materials.

$\left(1.0 \times 10^{23} \mathrm{eV} \mathrm{cm}^{-2}\right)$, panel $(b)$ shows a very strong modification of the structure in which $L_{\mathrm{a}}$ is $2.9 \mathrm{~nm}$ and long graphitic fibers are seen. In general, the average length of graphene layers $L_{\mathrm{a}}$ of the collected materials increases with the the exposure time of the UV irradiation. It varies from $1.82 \mathrm{~nm}$ for the sample irradiated for $40 \mathrm{~h}\left(5.2 \times 10^{22} \mathrm{eV} \mathrm{cm}^{-2}\right.$ of irradiation $)$ as seen in the panel $(c)$ to $2.2-2.5 \mathrm{~nm}$ for samples irradiated for $72 \mathrm{~h}\left(1.0 \times 10^{23} \mathrm{eV} \mathrm{cm}^{-2}\right)$ as seen in panels $(d),(e)$ and $(f)$. Partially, the small fiber structures may be combined to form longer fibers. Short and long graphitic fibers (or individual fiber) can be observed frequently with the UV irradiation as written down in the fourth column in Table 2. The small fullerene-like carbon particles are still visible between the graphite fibers because of shielding effects due to the limited penetration of the UV irradiation. Finally we can conclude that samples have similar graphene lengths (sizes) after UV irradiation.

\subsection{Hydrogen content and $s p^{3} / s p^{2}$ ratio in HAC materials}

\subsubsection{Hydrogen content and $\mathrm{sp}^{3} / \mathrm{sp}^{2}$ ratio of non-irradiated HAC materials}

The laser power density can partially change the electronic structure of the produced carbonaceous nanoparticles by influencing the temperature in the condensation zone. Samples have been 
Table 2. The average length of the graphene layers of HAC materials before and after the UV irradiation.

\begin{tabular}{|c|c|c|c|c|}
\hline \multirow{2}{*}{ S\# } & \multirow{2}{*}{$\begin{array}{l}\text { UV dose } \\
\left(\mathrm{eV} \mathrm{cm}^{-2}\right)\end{array}$} & \multicolumn{2}{|c|}{$L_{\mathrm{a}}(\mathrm{nm})$} & \\
\hline & & Average & Largest & \\
\hline S1 original & $\ldots$ & 0.42 & 2.7 & \\
\hline S2 original & $\ldots$ & 0.45 & 3.1 & \\
\hline S3 original & $\ldots$. & 0.43 & 1.8 & \\
\hline S26 original & $\ldots$. & 0.72 & 2.5 & \\
\hline S3 (grid irradiated for $23 \mathrm{~h})$ & $3.0 \times 10^{22}$ & 1.72 & 9.8 & a few fibers between $\mathrm{AC}$ structures up to $15 \mathrm{~nm}$ (2-3 nm thickness) \\
\hline S3 (grid irradiated for $72 \mathrm{~h}$ ) & $1.0 \times 10^{23}$ & 2.9 & 15.1 & more long-ordered fibers between AC structures (12 nm thickness) \\
\hline $\mathrm{S} 3$ irradiated for $40 \mathrm{~h}$ & $5.2 \times 10^{22}$ & 1.82 & 6.6 & long-ordered fibers between AC structures (5.8 nm thick) \\
\hline $\mathrm{S} 4$ irradiated for $72 \mathrm{~h}$ & $1.0 \times 10^{23}$ & 2.2 & 8.0 & individual fiber between $\mathrm{AC}$ structures \\
\hline $\mathrm{S} 7$ irradiated for $72 \mathrm{~h}$ & $1.0 \times 10^{23}$ & 2.3 & 5.1 & individual fiber between AC structures \\
\hline S8 irradiated for $48 \mathrm{~h}$ & $6.3 \times 10^{23}$ & 1.7 & 8.3 & a few fibers between $\mathrm{AC}$ structures up to $10 \mathrm{~nm}$ ( $1.5 \mathrm{~nm}$ thick) \\
\hline S26 irradiated for $72 \mathrm{~h}$ & $1.0 \times 10^{23}$ & 2.5 & 8.3 & long fibers between $\mathrm{AC}$ structures \\
\hline
\end{tabular}

produced by using low laser power density $\left(2 \times 10^{8} \mathrm{~W} \mathrm{~cm}^{-2}\right)$, which is close to what Jäger et al. (2008) used, and by high laser power density (as much as possible, $(1.4-3.1) \times 10^{10} \mathrm{~W} \mathrm{~cm}^{-2}$ ). The high laser power density allows the formation of the strong -C-H stretching band around $3.4 \mu \mathrm{m}$ with a high mass absorption coefficient $(\kappa)$ which is much higher than that in case of using the low laser power density. The hydrogen content which is represented by the $\mathrm{H} / \mathrm{C}$ ratio can be spectroscopically determined from the integrated mass absorption coefficient $\left(k_{\mathrm{CH}}\right)$ of the $\mathrm{C}-\mathrm{H}$ stretching band.

For this we used the formulae given in the appendix of Mennella et al. (2002), which are based on H/C-dependent absorption cross-section $(\sigma)$ of $\mathrm{C}-\mathrm{H}$ bonds determined by Jacob \& Unger (1996). Both $\left(k_{\mathrm{CH}}\right)$ and the $\mathrm{H} / \mathrm{C}$ ratio are shown in Table 1.

$\mathrm{The} \mathrm{sp}^{3} / \mathrm{sp}^{2}$ ratio can be estimated by using the expression,

$\frac{\mathrm{sp}^{3}}{\mathrm{sp}^{2}}=\frac{6 X_{\mathrm{H}}-1}{8-13 X_{\mathrm{H}}}$

where $X_{\mathrm{H}}=\mathrm{H} / \mathrm{C} /(1+\mathrm{H} / \mathrm{C})$. Equation (6) had been derived by Angus \& Jansen (1988) using an approach of the structure of a HAC material in terms of random covalent networks modeled by Phillips (1979). The $\mathrm{sp}^{3} / \mathrm{sp}^{2}$ ratio is limited by Eq. (6) according to the $X_{\mathrm{H}}$ which is restricted to the range from 17 to $62 \%(\mathrm{H} / \mathrm{C}$ is ranging from 0.2 to 1.6 , respectively). This ratio is mainly growing in correlation with the hydrogen fraction. It is concluded that using the high laser power density to produce samples of a HAC material increases the fraction of carbon atoms and even small carbon molecules. These products being bonded with the plenty hydrogen can form single, double and triple forms resulting in an slight increase of the $\mathrm{sp}^{3}$ hybridization. Equation (6) does not consider carbon atoms having $\mathrm{sp}^{1}$ hybridization which is observed at about $3300 \mathrm{~cm}^{-1}$ (Fig. 4). If we take the $\mathrm{sp}^{1}$ hybridization into account, it is expected, according to Eq. (3) in Angus \& Jansen (1988), that the presence of the $\mathrm{sp}^{1}$ hybridization increases $\mathrm{sp}^{3} / \mathrm{sp}^{2}$ ratios. However, in this case a derivation of the $\mathrm{sp}^{3} / \mathrm{sp}^{2}$ ratios is not explicitly possible.

\subsubsection{Hydrogen content and $\mathrm{sp}^{3} / \mathrm{sp}^{2}$ ratio of irradiated $\mathrm{HAC}$ materials}

The HAC material loses much hydrogen during the irradiation process leading to the rearrangement in the internal atomic and electronic structures. As illustrated from the left panels of Fig. 4, the mass absorption coefficient of the spectra of S4, S24 and $\mathrm{S} 26$ at the $\mathrm{C}-\mathrm{H}$ stretching band position in the $3000-2800 \mathrm{~cm}^{-1}$ $(3.33-3.57 \mu \mathrm{m})$ range has been reduced after irradiation while the $\equiv \mathrm{C}-\mathrm{H}$ stretching band at $3300 \mathrm{~cm}^{-1}(3.03 \mu \mathrm{m})$ is completely removed. The estimated $\mathrm{H} / \mathrm{C}$ and $\mathrm{sp}^{3} / \mathrm{sp}^{2}$ ratios as shown in the right (top and bottom, respectively) panels of Fig. 4 decrease as a function of the UV dose. The structure of the samples S24 and S26 is changed much more effectively by UV irradiation than those of sample S4, even though S4 and S26 are irradiated for the same time. It is probable that $\mathrm{S} 4$ is getting some of the hydrogen content from the contamination (hydrocarbon molecules) of this sample because it contains more pores than the others. This contamination usually occurs after the deposition process once the samples are exposed to air. It has a strong effect on the samples possesing a low mass absorption coefficient. Apart from the contamination of the samples, the rate of decreasing the $\mathrm{H} / \mathrm{C}$ and $\mathrm{sp}^{3} / \mathrm{sp}^{2}$ ratios of $\mathrm{S} 24$ and $\mathrm{S} 26$, which have high mass absorption coefficients, is mostly the same. This means that the UV radiation can break up a larger amount of the $\mathrm{C}-\mathrm{H}$ bonds of the $\mathrm{HAC}$ materials possessing a high mass absorption coefficient than in HAC materials possessing a low mass absorption coefficient. We can conclude that the UV processing of HAC increases the carbon fraction relative to the hydrogen content, providing the possibility to change $\mathrm{sp}^{3}$ into $\mathrm{sp}^{2}$ hybridization and thereby to order the sites of $\pi$ bonds.

\subsection{Gap energy $\left(E_{g}\right)$ of HAC materials from UVIVIS spectroscopy}

For an amorphous semiconductor material such as a HAC material, optical absorption measurements in the NIR to the FUV range provide information about the electronic structure of this material such as the optical bandgap energy. This gap energy $E_{\mathrm{g}}$ can be estimated from the Tauc relation

$\sqrt{\alpha E}=B\left(E-E_{\mathrm{g}}\right)$

where $\alpha$ is the linear attenuation coefficient, $E$ is the photon energy, and $B$ is a constant. $E_{\mathrm{g}}$ and the slope $B$ are related to the range of the aromatic cluster size of the HAC materials (Robertson \& O'Reilly 1987). This relation is mostly used in the laboratory to determine the gap energy of materials prepared both by thin-film or particle deposition. We note that the gap energy determined from the Tauc relation exhibits some uncertainties. This approach applied to a particulate material can lead 

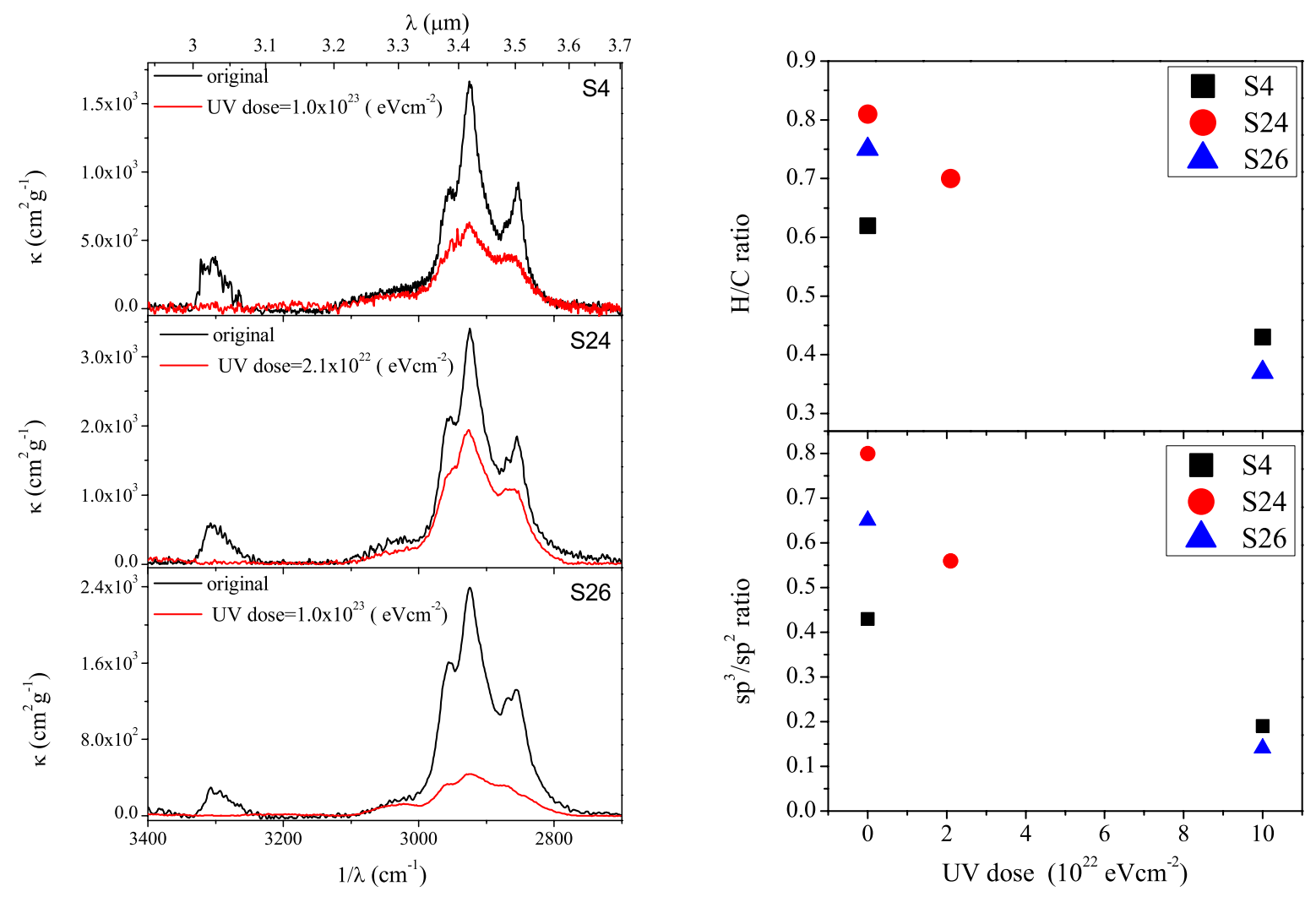

Fig. 4. Hydrogen content and $\mathrm{sp}^{3} / \mathrm{sp}^{2}$ ratio as a function of irradiated HAC materials.

to different results compared to continuous films, because it is based on a proportionality between $\alpha$ and the imaginary part of the dielectric function, which is strictly valid only for a continuous material. However, we have checked by simulation of thinfilm vs. particle absorption spectra (using Mie theory for the latter) that these uncertainties are in the range of $\Delta E_{\mathrm{g}}= \pm 0.2 \mathrm{eV}$ only for a hydrogenated material (we have used the $\mathrm{ACH} 2$ data by Zubko et al. 1996).

The Tauc approach gives a good parameterization of the HAC absorption edge in the VIS range (Mennella et al. 1995). In Fig. 5, the shown spectra are characterized by increasing absorption toward the FUV band. Instead of $\alpha$, we have used the $\kappa$ spectra which are related to $\alpha$ via $\kappa=\alpha / \rho$, where $\rho$ is the mass density. Whether using $\alpha$ or $\kappa$ is not important for the determination of $E_{\mathrm{g}}$ because it influences only the $y$-axis value of the Tauc plot but not the slope towards smaller energies. The corresponding materials have different values of Tauc $E_{\mathrm{g}}$ given by the intersection of the extrapolation of the curves with the energy axis.

From the gap energies, other structural properties can also be estimated according to the approach of Robertson (1986), such as the average length of the graphene layers $\left(L_{\mathrm{a}}\right)$ and the number of six-fold rings $(M)$ inside aromatic clusters according to the following relation

$$
E_{\mathrm{g}} \approx-\frac{2 \beta}{\sqrt{M}} \approx \frac{6}{\sqrt{M}} \mathrm{eV} \approx \frac{7.7}{L_{\mathrm{a}}} \mathrm{eV}
$$

where $L_{\mathrm{a}}$ has to be taken in $\AA$, and $\beta=-2.9 \mathrm{eV}$ is the Huckel parameter. We should note here that the relation for $M$, which is based on the Huckel model, should give the number of aromatic rings for the largest clusters that are significantly contributing to the electronic density of states. The relation for $L_{\mathrm{a}}$, in contrast, is an empirical relation for the average crystallite sizes such as the ones determined by Raman or X-ray measurements.

\subsubsection{Gap energy $\left(E_{g}\right)$ of non-irradiated HAC materials}

Because of the production of many $\mathrm{C}-\mathrm{H}$ bonds with the high laser power and the associated change of the $\mathrm{sp}^{3} / \mathrm{sp}^{2}$ ratios, the gap energy is expected to increase in the range of the semiconductor materials (HAC materials). For samples S1-S11 and S14-S23 (Table 1), the values of the gap energy have been obtained. They are in the range between $1.1-1.55 \mathrm{eV}$ for samples S1-S11 and 1.65-2.15 for samples S14-S23. There is a distinguishable difference in the gap energy between samples S1-S11 produced with using low laser power density and samples S14S23 produced with using high laser power density. As a result, the gap energy increases as the hydrogen content increases. In addition, the corresponding peak positions in the FUV band are shifted toward longer wavelengths (see Sect. 3.4). As determined from Eq. (8), the value of $M$ is ranging inversely with the gap energy from 14 to 27 at the low values of the hydrogen content and from 7 to 12 at the higher values of the hydrogen content. These aromatic cluster sizes are clearly larger than the average cluster sizes determined from HRTEM (3-8 rings corresponding to cluster diameters of 0.42 to $0.72 \mathrm{~nm}$, see upper part of Table 2). This is reasonable because clusters larger than the average, allowing for the lowest significant energetic $\pi$-electronic transitions, should limit the optical gap. On the other hand, this means that the largest graphene layers seen in our HAC materials by HRTEM (48 to 144 rings corresponding to diameters of $1.8-3.2 \mathrm{~nm}$ ) are probably too rare for contributing significantly to the density of states (with respect to the Tauc gap). 


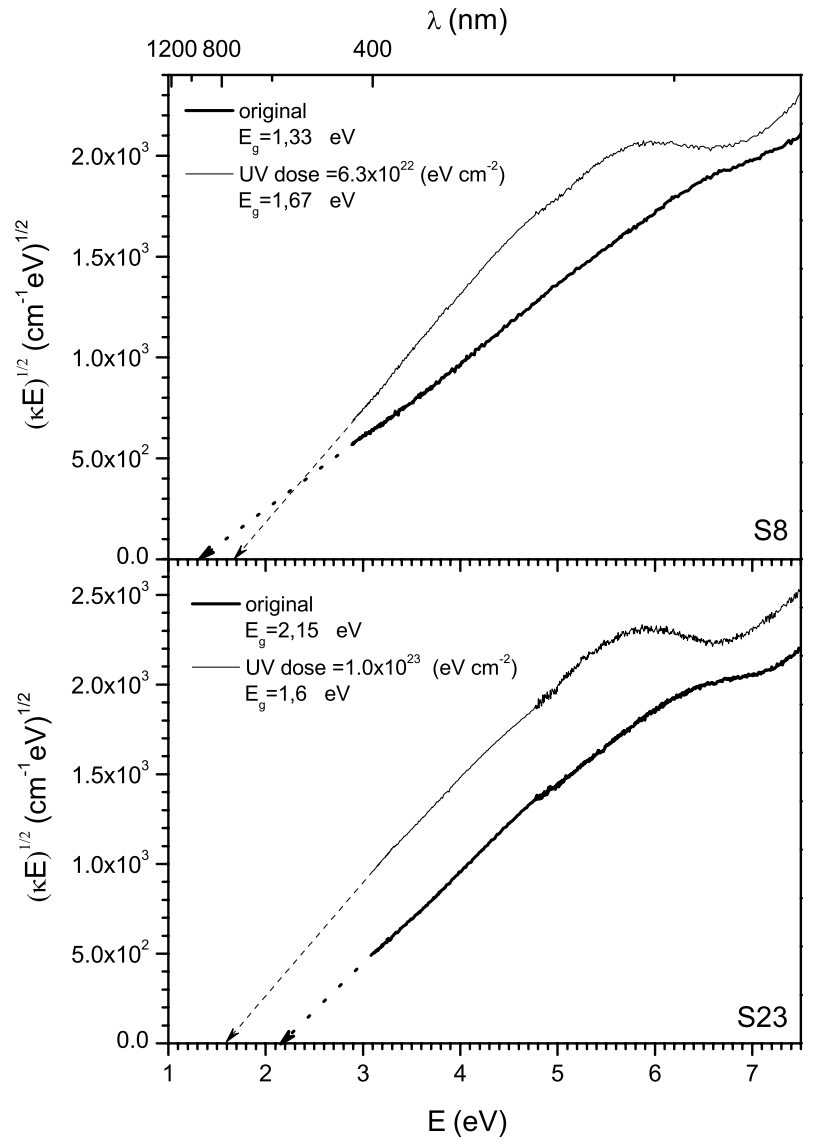

Fig. 5. Gap energy of HAC materials (S4, S8, S21 and S23) determined by using Tauc relation as a function of the UV irradiation.

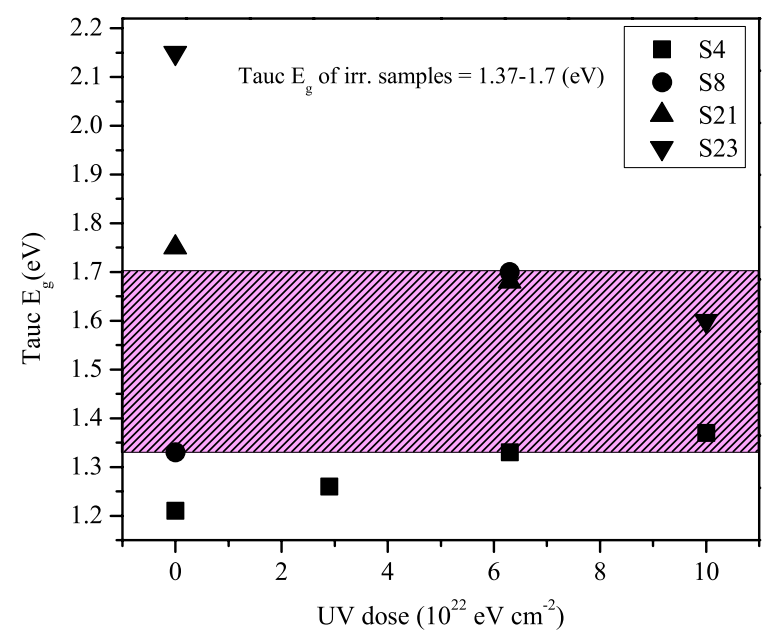

Fig. 6. Gap energy of irradiated HAC materials (S4, S8, S21 and S23) compared to those of the original materials. The shaded area gives the approximate range to which the gap energy converges after irradiation.

The value of $L_{\mathrm{a}}$ determined from Eq. (8) is ranging from 0.36 to $0.7 \mathrm{~nm}$. This is in approximate agreement with the average $L_{\mathrm{a}}$ values determined from the HRTEM analysis $(0.42-0.72 \mathrm{~nm})$ as expected.

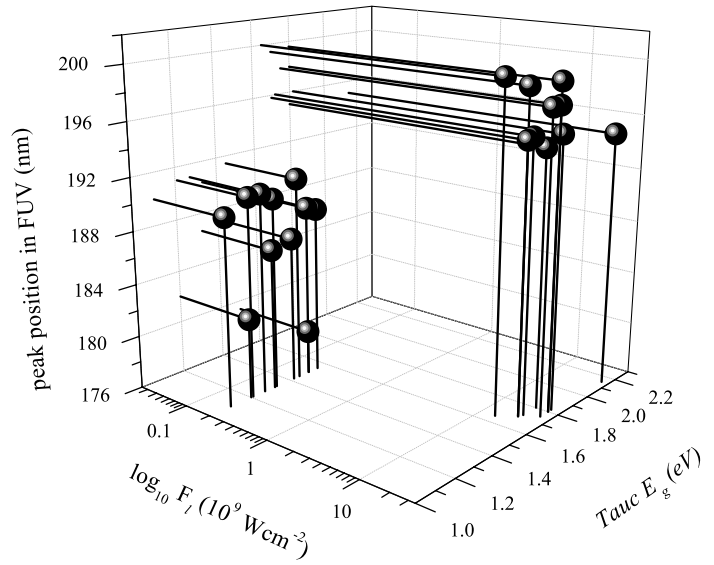

Fig. 7. Gap energy of HAC materials samples (S1-S11 and S14-S23) determined by using Tauc relation and its correlation to the peak position in FUV as a function of the laser power density.

\subsubsection{Gap energy $\left(E_{g}\right)$ of irradiated HAC materials}

In fact, the gap energy of HAC materials has differently been affected by the UV irradiation as given in Table 1 . Figure 5 shows that the gap energy of S8 having a low hydrogen content is changing in the unexpected direction. It increases from 1.33 to $1.67 \mathrm{eV}$ with increasing UV irradiation dose. On the other hand for S23 having a high hydrogen content, the gap energy behaves as expected. It decreases from 2.15 to $1.6 \mathrm{eV}$, with UV irradiation at different doses. Figure 6 shows that the gap energy of irradiated HAC materials is located around the value $1.535 \pm 0.165 \mathrm{eV}$ whatever the Tauc $E_{\mathrm{g}}$ value of the non-irradiated HAC materials is.

The evolution of both $M$ and $L_{\mathrm{a}}$ is inversely related to the variation of Tauc $E_{\mathrm{g}}$ as mentioned above. After UV irradiation, $M$ can be ranging from 11 to 17 rings inside the cluster of irradiated materials, taking into account that these values are the integer values of Eq. (8). The value $L_{\mathrm{a}}$ of irradiated HAC materials can also be estimated by using the same equation, and it is ranging from 0.46 to $0.56 \mathrm{~nm}$. Irradiated HAC materials are converted towards certain values of graphene layer lengths. These values are in clear contradiction with the $L_{\mathrm{a}}$ values derived from HRTEM analysis (see lower part of Table 2). These average values of $L_{\mathrm{a}}$ ranging from 1.7 to $2.9 \mathrm{~nm}$ would correspond to gap energy values in the range 0.25 to $0.45 \mathrm{eV}$. The finding that the measured gap energies do not reflect the graphitization of the samples observed by HRTEM is very likely due to the application of the Tauc formula to a case where $\alpha$ is not proportional to the imaginary part of the dielectric function. Similar simulations using Mie theory as mentioned above (see Sect. 4.1) indeed show that the Tauc approach applied to absorption spectra of more graphitic particles (here we used the BE data from Zubko et al. 1996) can considerably overestimate the optical gap energy. These simulations included scattering effects taking the increased particle size after irradiation into account. Thus, we think that the measured convergence of the $E_{\mathrm{g}}$ values to values around $1.5 \mathrm{eV}$ is an artificial effect and that the true optical gap energies are rather in the range 0.25 to $0.45 \mathrm{eV}$. 

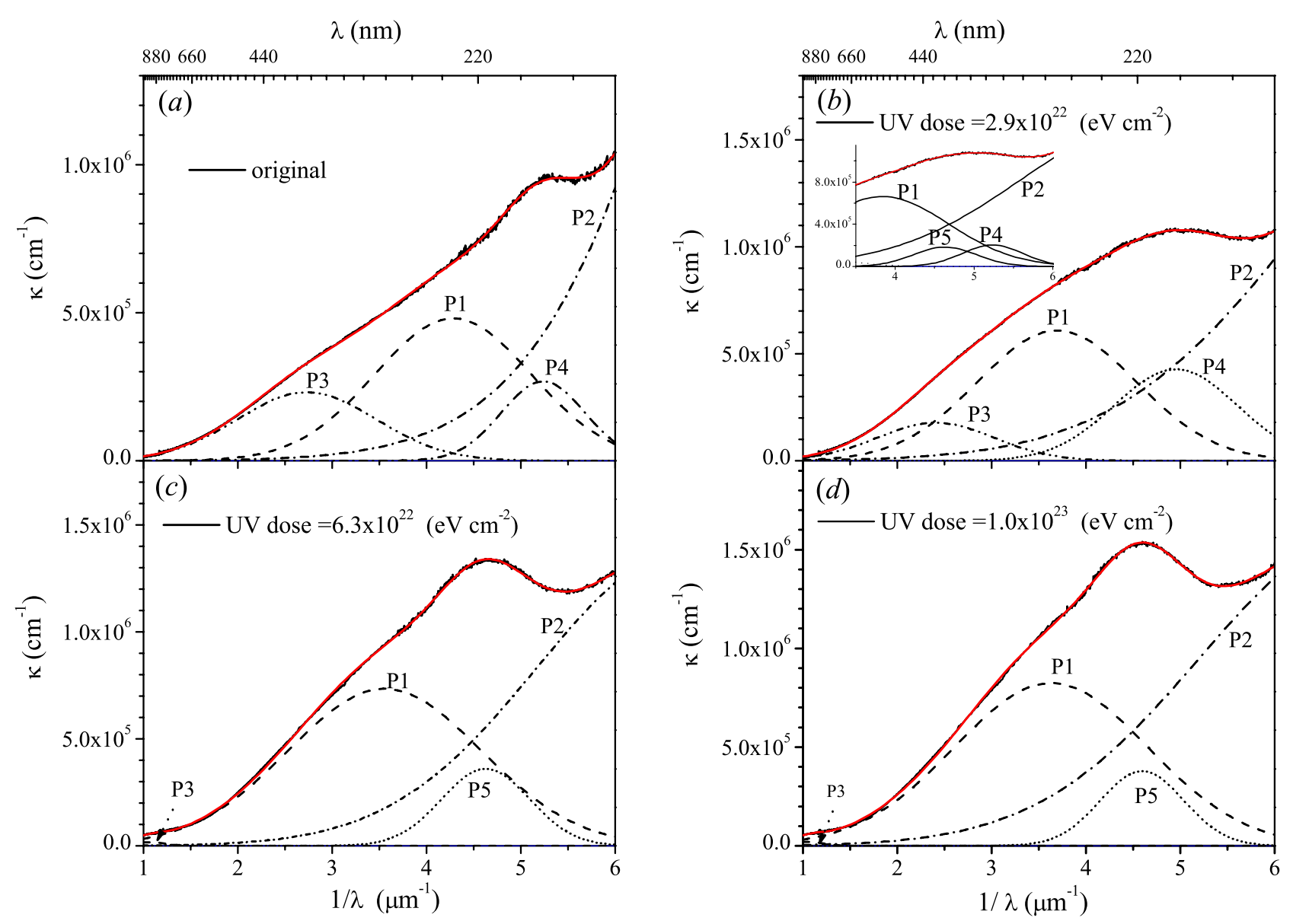

Fig. 8. Deconvolution of the absorption spectra of S4; a) for the original spectrum, b), c) and d) for exposures to UV doses of $2.9 \times 10^{22}, 6.3 \times 10^{22}$ and $1.0 \times 10^{23}\left(\mathrm{eV} \mathrm{cm}^{-2}\right)$, respectively. The inset in the panel b) shows that the rise might be due to the existence of P4 and P5 (small new band at $\left.4.6 \mu \mathrm{m}^{-1}\right)$.

\subsection{The spectroscopy in the FUV-NIR range of irradiated HAC materials}

There are different transitions of electrons taking place within atomic and molecular orbitals of the carbonaceous materials. The $n-\pi^{*}$ and $\pi-\pi^{*}$ transitions are in the wavelength range from 250 to $600 \mathrm{~nm}\left(4\right.$ to $\left.1.67 \mu \mathrm{m}^{-1}\right)$ and from 200 to $500 \mathrm{~nm}$ (5 to $\left.2 \mu \mathrm{m}^{-1}\right)$, respectively. Others $\left(\sigma-\sigma^{*}\right.$ and $\left.n-\sigma^{*}\right)$ occur at $\lambda<200 \mathrm{~nm}$ and $160<\lambda>260 \mathrm{~nm}$, respectively. According to Llamas-Jansa et al. (2007) for a HAC material produced by pulsed-IRLP, the spectrum from the FUV to the NIR range can be parameterized into different Gaussian contributions (with different peak positions). They had considered four peaks that can be observed in this range. The first (hereafter, P1) and second (hereafter, P2) peaks were representing the central $\pi-\pi^{*}$ and $\sigma-\sigma^{*}$ transitions, respectively, while the third (hereafter, P3) peak was considered as the $\pi-\pi^{*}$ transitions at longer wavelengths occurring due to the structural disorder. The fourth peak (hereafter, P4) had been centered in the range from 5.1 to $5.2 \mu \mathrm{m}^{-1}$ and it was considered as accounting for an additional $n-\sigma^{*}$ transition feature resulting from the attached $\mathrm{O}$-containing molecular groups such as $\mathrm{OH}$ groups. These $\mathrm{O}$ bearing molecules could be due to the air exposure of the samples.

The spectra of our original materials as shown in Fig. 9 (panel $a$ ) are similar to those of the above-mentioned IRLP materials. As shown in Fig. 7, the position of P4 in our spectra is related to the laser power density (or to the gap energy). It is in the range from 5.0 to $5.3 \mu \mathrm{m}^{-1}$ shifting to the longer wavelengths with increasing the gap energy. If it is true that this peak originates from oxygen-bearing species, this trend could be produced by the different porosities of the high laser power and low laser power materials, respectively.

In Fig. 8, we will show the deconvolution into Gaussians for one representative example (S4). The spectrum of the original sample (panel $(a)$ ) can be parameterized by using Gaussian fitting to four peaks similar to that of Llamas-Jansa et al. (2007). These peaks have a small displacement of the peak positions compared to those of Llamas-Jansa et al. (2007). As shown in panels $(b),(c)$ and $(d)$, Gaussian fitting of the spectra of the irradiated sample shows a dramatic change of the peak positions and widths. This change is due to the modification of the structural properties $\left(L_{\mathrm{a}}, \mathrm{H} / \mathrm{C}\right.$ ratio and $\mathrm{sp}^{3} / \mathrm{sp}^{2}$ hybridization ratio) which indicate that the processed-HAC materials become much more aromatic and ordered materials. Panel $(b)$ shows the spectrum of the sample which has been irradiated for a relatively short time $(22 \mathrm{~h})$. The variation of $\mathrm{P} 4$ in this spectrum compared to the non-processed sample is not clear. This peak could be shifted toward smaller wavenumbers $\left(4.95 \mu \mathrm{m}^{-1}\right)$. As tabulated in the Table $2\left(\mathrm{~S} 3_{\text {grid }}\right)$, the value $L_{\mathrm{a}}$ becomes longer $(1.7 \mathrm{~nm})$, at approximately the same UV irradiated time $(23 \mathrm{~h})$, relative to that of non-processed HAC materials, which could produce this shift. It is also possible that the band at $4.95 \mu \mathrm{m}^{-1}$ is due to a combination of $\mathrm{P} 4$, which becomes somewhat smaller, with a new small 
peak (we call it as P5) which is due to the UV irradiation. In this case the new band would be centered around $4.63 \mu \mathrm{m}^{-1}$ (see the inset in the panel $(b)$ ). After longer UV irradiation (panels $(c)$ and $(d)$ ), the peak P3 becomes very small and shifts to the NIR, whereas the new peak P5 at 4.62 and $4.595 \mu \mathrm{m}^{-1}$, respectively, becomes much stronger and sharper, and very close to the interstellar UV band called UV bump at $4.6 \mu \mathrm{m}^{-1}(217.5 \mathrm{~nm})$. Since the graphene layers become even longer (2.2 to $2.9 \mathrm{~nm}$ ) with the higher UV irradiation dose, the spectroscopic measurements result in a big variation of $\mathrm{P} 1, \mathrm{P} 4$ and $\mathrm{P} 5$ components, in particular their positions. With the UV irradiation the position P5 becomes more stable at $4.6 \mu \mathrm{m}^{-1}$. We have to consider that, for larger particles produced by irradiation, the measured spectra contain a contribution of scattering losses. For this kind of larger HAC particles, the scattering contributions to the extinction spectra has been measured and also calculated by LlamasJansa et al. (2007). These scattering contributions appear to be quite spectrally flat and should not significantly influence the spectral features.

As a result, the structural modification is related to the variation in the electronic density of states, in particular to the $\pi-\pi^{*}$ transitions. This indicates that the distinguishable UV band at $4.6 \mu \mathrm{m}^{-1}$ can be produced by the UV irradiation of carbonaceous nanoparticles in interstellar space as proposed by Mennella et al. (1996). It probably results from $\pi-\pi^{*}$ electronic transitions due to the existence of the aromatic structures motivated by the UV radiation within the HAC material. Table 4 summarizes how the Gaussian fitting parameters P1, P4 and P5 and their widths (W1, W4 and W5) have changed with the UV irradiation of all irradiated samples. In this table, we are taking only into account the parameters P1, P4 and P5 because they are mainly describing the profile of the spectrum. For the spectra of irradiated samples, $\mathrm{P} 2$ has no strong effect for the fitting process.

\section{Astronomical implications}

According to the recent studies by Cardelli et al. (1989), Fitzpatrick (1999, hereafter F99), and Fitzpatrick \& Massa (2007, hereafter FM07) in the FUV-NIR wavelength range $(125 \mathrm{~nm}$ to $3500 \mathrm{~nm})$, the interstellar extinction given as the quantity $A(\lambda) / A(V)$ consists of two components. The first one represents a linear background. The second is the Lorentzianlike $4.6 \mu \mathrm{m}^{-1}(217.5 \mathrm{~nm})$ bump. The trend of the extinction curve from the FUV through the NIR in our own galaxy is well characterized by the single parameter $R(V)$ which represents the total to selective extinction ratio. The value of $R(V)$ is between 2.5 and 5.8 in different sight lines (Cardelli et al. 1989; Valencic et al. 2004). Fitzpatrick (2004) found that the strength of the $4.6 \mu \mathrm{m}^{-1}$ bump is related to the $R(V)$. The strong bumps occur along sight lines which have medium values of $R(V)$.

Figure 9 shows the extinction cross section per number of carbon atoms $\left(C_{\text {ext }} / N_{\mathrm{C}}\right)$ of the laboratory spectra of HAC materials (S4, S7, S8, S21 and S23) before $(a)$ and after $(b)$ UV irradiation. In panel $(c)$, comparable curves of the extinction cross section per hydrogen atom $\left(C_{\text {ext }} / N_{\mathrm{H}}\right)$ of $\mathrm{F} 99$ and FM07 (at $R(V)=$ 3.1 and 3.001, respectively) in addition to those of some objects chosen from 328 galactic extinction curves (FM07) (with different values of $R(V)$ ) are shown. The dotted curve represents the average value at $R(V)=3.001$ of galactic diffuse extinction cross section curves of 243 stars with $2.6<R(V)<3$.6. The curves in panel $(c)$ are calculated by using the parameters taken from FM07 and from F99. The band position of the $4.6 \mu \mathrm{m}^{-1}$ bump is shown by the vertical dotted line. The comparison demonstrates that there is an agreement of the band positions of the laboratory
Table 3. Gaussian fitting parameters of spectra before and after UV irradiation.

\begin{tabular}{|c|c|c|c|c|c|}
\hline \multirow[t]{2}{*}{ S\# } & \multirow{2}{*}{$\begin{array}{l}\mathrm{P}^{a} / \mathrm{W}^{b} \\
\left(\mu \mathrm{m}^{-1}\right)\end{array}$} & \multirow[t]{2}{*}{ Original } & \multicolumn{3}{|c|}{$\mathrm{UV}$ dose $\left(\times 10^{23} \mathrm{eV} \mathrm{cm}^{-2}\right)$} \\
\hline & & & 0.28 & 0.63 & 1.0 \\
\hline \multirow{6}{*}{ S4 } & $\mathrm{P} 1$ & 4.28 & 3.69 & 3.55 & 3.62 \\
\hline & W1 & 1.95 & 1.98 & 2.41 & 2.41 \\
\hline & $\mathrm{P} 4$ & 5.24 & $4.95^{c}(5.24)^{d}$ & $\ldots$ & $\ldots$ \\
\hline & W4 & 1.04 & $1.53^{c}(0.90)^{d}$ & $\ldots$. & $\ldots$ \\
\hline & $\overline{\mathrm{P} 5}$ & $\ldots$ & $4.63^{d}$ & 4.62 & 4.595 \\
\hline & W5 & $\ldots$. & $0.94^{d}$ & 1.1 & 1.04 \\
\hline \multirow{6}{*}{ S7 } & P1 & 4.09 & & & 4.02 \\
\hline & W1 & 2.18 & & & 2.38 \\
\hline & $\mathrm{P} 4$ & 5.223 & & & $\ldots$ \\
\hline & W4 & 1.19 & & & $\ldots$ \\
\hline & P5 & $\ldots$. & & & 4.607 \\
\hline & W5 & $\ldots$. & & & 1.041 \\
\hline \multirow{6}{*}{ S8 } & P1 & 4.36 & & 3.81 & \\
\hline & W1 & 2.89 & & 2.27 & \\
\hline & $\mathrm{P} 4$ & 5.252 & & $\cdots$ & \\
\hline & W4 & 1.27 & & $\ldots$ & \\
\hline & P5 & $\ldots$. & & 4.69 & \\
\hline & W5 & $\ldots$. & & 1.15 & \\
\hline \multirow{6}{*}{$\mathrm{S} 21$} & P1 & 4.12 & & 3.79 & \\
\hline & W1 & 2.47 & & 2.18 & \\
\hline & $\mathrm{P} 4$ & 5.057 & & $\ldots$. & \\
\hline & W4 & 1.14 & & $\ldots$ & \\
\hline & $\overline{\mathrm{P} 5}$ & $\ldots$ & & 4.639 & \\
\hline & W5 & $\ldots$ & & 1.12 & \\
\hline \multirow{6}{*}{ S23 } & P1 & 4.25 & & & 3.53 \\
\hline & W1 & 2.29 & & & 1.93 \\
\hline & P4 & 5.13 & & & $\cdots$ \\
\hline & W4 & 1.15 & & & $\ldots$ \\
\hline & P5 & $\ldots$ & & & 4.608 \\
\hline & W5 & $\ldots$ & & & 1.11 \\
\hline
\end{tabular}

Notes. ${ }^{(a)}$ the position of peaks (P1, P4 and P5). ${ }^{(b)}$ the width of peaks (W1, W4 and W5). ${ }^{(c)}$ If we consider only one peak shifted toward longer wavelength. ${ }^{(d)}$ If we consider that there is a combination of two peaks (P4 and P5).

measurements after the UV irradiation with those of the extinction curves of the diffuse interstellar medium (DISM).

Figure 10 shows the comparison of the UV bump at $4.6 \mu \mathrm{m}^{-1}$ (P5) extracted from the spectra of irradiated samples with those extracted from interstellar extinction cross section curves mentioned above by subtracting the linear component. For the laboratory spectra, the peak P5 has been fitted by Gaussian fitting as shown in Sect. 3.4 which allowed a distinguishable fitting of spectra in contrast to Lorentzian fitting, even though the bump in the interstellar medium has a Lorentzian shape. For comparison between the laboratory data and those of the astronomical data of F99 and FM07, the parameters of this bump including the band position and width in addition to the ratio of the carbon to hydrogen column density $\left(N_{\mathrm{C}} / N_{\mathrm{H}}\right)$ required to produce a feature of the observed strength in space have been compared in Table 4 to the standard values of the DISM (F99). In the laboratory results, the position of this band shifts toward longer wavelengths from 4.69 to $4.595 \mu \mathrm{m}^{-1}$ with increasing the dose of UV irradiation from 21 to $33 \%$ of D (D is the fluence (UV dose) expected during the life time of a diffuse interstellar cloud), respectively. This variation is in good agreement with the model done by Mennella et al. (1998). Their approach depends on the different degree of UV processing of hydrogenated carbon grains and predict peak 


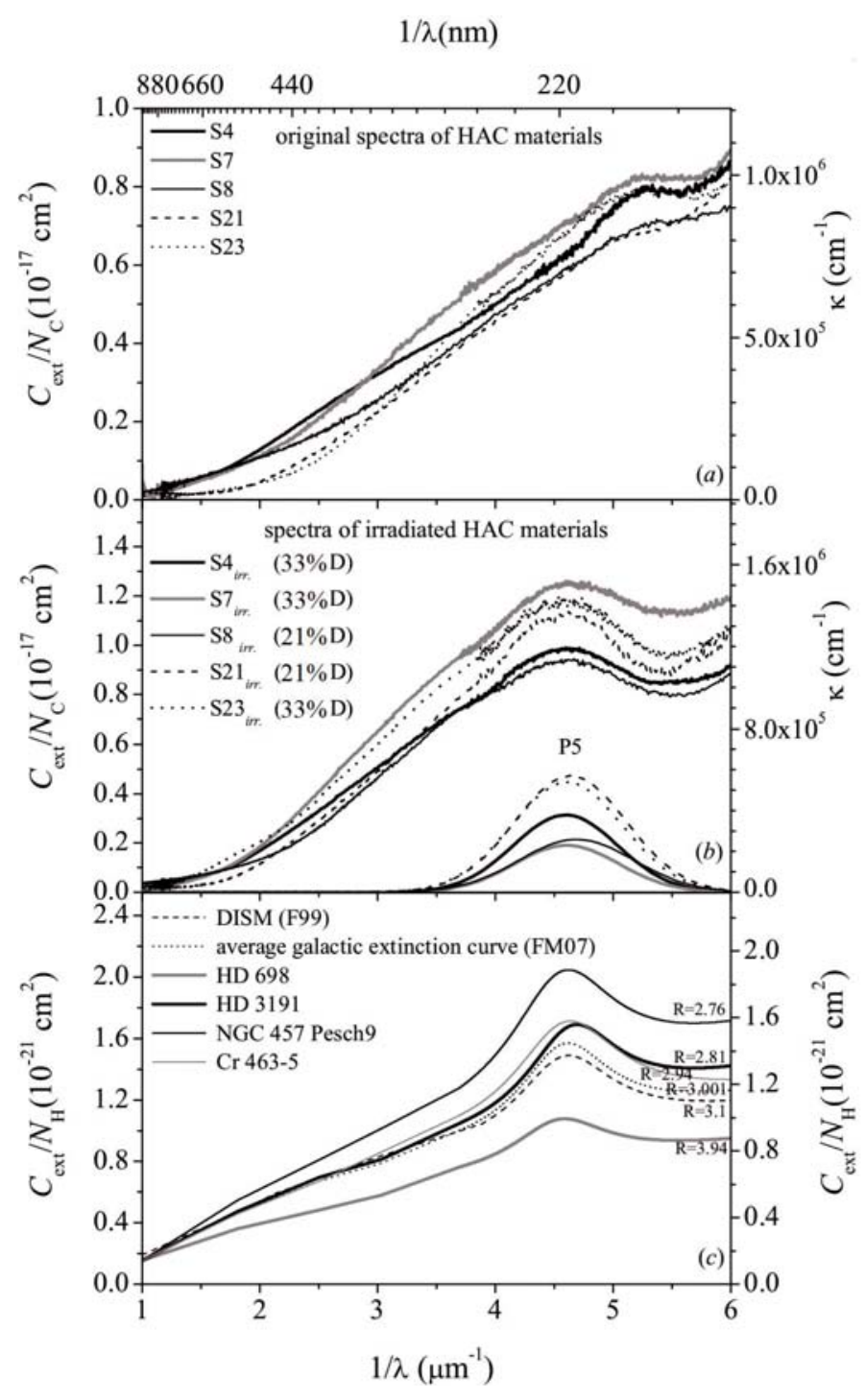

Fig. 9. The Panel a) shows the original spectra of S4, S7, S8, S21 and S23 before UV irradiation while the panel b) shows the spectra of S4, S7, S8, S21 and S23 after UV irradiation. In panel c), the interstellar extinction curves of DISM (Fitzpatrick 1999) and the average galactic extinction curve, as well as for the objects HD 698, HD 3191, NGC 457 Pesch9 and Cr 463-5 (Fitzpatrick \& Massa 2007) are shown. The curves labeled P5 in panel b) are the extracted UV bump profiles around $4.6 \mu \mathrm{m}^{-1}$.

positions of the UV bump by extrapolating the results of Mennella et al. (1996) up to the expected UV dose in the diffuse cloud time-scale. For the doses that we have reached experimentally, the measured peak positions are very close to the predicted ones.

There is a good agreement for these samples with the highest irradiation dose, at which the central wavelength $\left(\lambda_{0}^{-1}\right)$ of the bump is relatively constant. The column number 4 in Table 4 shows a small deviation $\left(\Delta \lambda_{0}^{-1}=\lambda_{0}^{-1}-\left(\lambda_{0}^{-1}\right)_{\text {DISM }}\right)$ at the lowest values of the UV irradiation dose. This deviation is acceptable in comparing with those values of some observed objects. For diffuse medium environments, the full width at half maximum (FWHM) of UV bump varies considerably from 0.77 to $1.25 \mu \mathrm{m}^{-1}$ or from 36 to $60 \mathrm{~nm}$ (Fitzpatrick \& Massa 1986). The FWHM values of P5 (the third column of Table 4) lie in this extent and it seems that an increase of the UV irradiation dose makes this peak narrower and better fitting with the

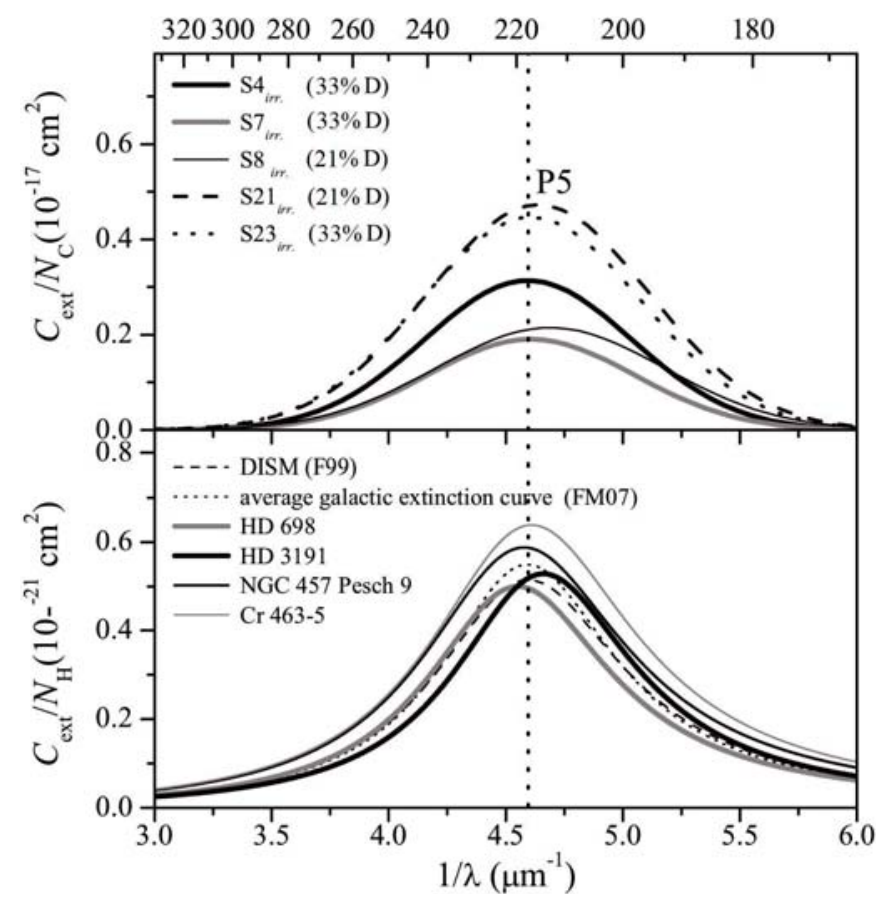

Fig. 10. The upper panel shows the extracted component P5 $\left(4.6 \mu \mathrm{m}^{-1}\right)$ from spectra of irradiated HAC materials (S4, S7, S8, S21 and S23). The bottom panel shows the extracted bump $\left(4.6 \mu \mathrm{m}^{-1}\right)$ of the interstellar extinction curves of DISM (Fitzpatrick 1999) and the average galactic extinction curve, as well as towards the stars HD 698, HD 3191, NGC 457 Pesch9 and Cr 463-5 (Fitzpatrick \& Massa 2007).

Table 4. Parameters of the UV bump at $4.6 \mu \mathrm{m}^{-1}(217.5 \mathrm{~nm})$ in the spectra of irradiated HAC materials in comparison with those of the astronomical data.

\begin{tabular}{lccccc}
\hline \hline & $\begin{array}{c}\lambda_{0}^{-1} \\
\mu \mathrm{m}^{-1}\end{array}$ & $\begin{array}{c}F W H M \\
\mu \mathrm{m}^{-1}\end{array}$ & $\begin{array}{c}\Delta \lambda_{0}^{-1} \\
\mu \mathrm{m}^{-1}\end{array}$ & $\begin{array}{c}\Delta F W H M \\
\mu \mathrm{m}^{-1}\end{array}$ & $\begin{array}{c}N_{\mathrm{C}} / N_{\mathrm{H}}^{(a)} \\
\mathrm{ppm}\end{array}$ \\
\hline $\mathrm{S}_{\text {irr. }}(33 \% \mathrm{D})$ & 4.595 & 1.04 & -0.001 & 0.05 & 164 \\
$\mathrm{~S} 7_{\text {irr. }}(33 \% \mathrm{D})$ & 4.607 & 1.041 & 0.011 & 0.051 & 269 \\
$\mathrm{~S} 8_{\text {irr. }}(21 \% \mathrm{D})$ & 4.69 & 1.15 & 0.094 & 0.16 & 236 \\
$\mathrm{~S} 21_{\text {irr: }}(21 \% \mathrm{D})$ & 4.639 & 1.12 & 0.043 & 0.13 & 108 \\
$\mathrm{~S} 23_{\text {irr. }}(33 \% \mathrm{D})$ & 4.608 & 1.11 & 0.012 & 0.12 & 115 \\
DISM & 4.596 & 0.99 & 0.0 & 0.0 & $\ldots$. \\
average FM07 & 4.592 & 0.922 & -0.045 & -0.03 & $\ldots$. \\
HD698 & 4.551 & 0.96 & 0.04 & -0.05 & $\ldots$. \\
HD3191 & 4.636 & 0.94 & -0.018 & 0.08 & $\ldots$. \\
NGC457 P. 9 & 4.578 & 1.07 & -0.019 & 0.12 & $\ldots$. \\
Cr463-5 & 4.61 & 1.08 & 0.014 & 0.09 & $\ldots$. \\
\hline
\end{tabular}

Notes. ${ }^{(a)}$ The last column gives the required carbon abundance to match the band strength in diffuse ISM.

parameters derived from the astronomical data. A little increase of the width $\left(\triangle F W H M=F W H M-(F W H M)_{\text {DISM }}\right)$ of the bump appears in our processed-samples in comparing to that of the diffuse ISM as shown in the column number 5. Clustering of grains in the sample could cause a broadening of the bump (Schnaiter et al. 1998). Considering the results of the laboratory measurements, we should take into account that high carbon abundances would be required to match the strength of the DISM band. The required values of the $\left(N_{\mathrm{C}} / N_{\mathrm{H}}\right)$ ratio, shown in the last column 
of Table 4, which are inversely related to the strength of P5 in the laboratory spectra of irradiated HAC materials, are not well correlated with the irradiation dose. The carbon abundance locked in these materials would be varying between 108 and 269 ppm. This is very close to what Schnaiter et al. (1998) found.

As illustrated by the upper panel in Fig. 10, UV irradiation can more effectively produce rather high amplitude of the UV bump for samples (S21 and S23) having originally high hydrogen content and higher gap energy. We do not know actually how the hydrogen content precisely influence the interaction with the UV radiation. The intensity of the UV bump in our irradiated spectra is smaller than that found by Mennella et al. (1996). We attribute this to the inhomogeneity of the irradiated material, as shown by HRTEM in Fig. 3 (e.g. panel c). By this we do not mean a lateral inhomogeneity, because the measured area (with a diameter $1 \mathrm{~mm}$ ) is always smaller than the irradiated area (with a diameter $1.5 \mathrm{~mm}$ ). The inhomogeneity of irradiated particles could occur due to the shielding by the outer parts of the sample, in front of the incident UV beam on the samples, that might protect the inner parts of the sample or at least attenuate the UV beam penetrating inside the sample.

The carbon abundance relative to hydrogen in the ISM is $225 \pm 55$ ppm (Snow \& Witt 1995). It includes $140 \pm 20$ ppm (Cardelli et al. 1996) in the gas phase, leaving only $85 \pm 55 \mathrm{ppm}$ available for the dust. The last value may be too small to produce the UV bump based on our materials, although some variability of the carbon abundances in the gas-phase can be observed through different lines of sight in the ISM (Sofia \& Parvathi 2009). This fact has been noted by other authors before and has been named the carbon crisis in ISM (Kim \& Martin 1996). After the results obtained in this work, we have to consider the relation between carbon abundances in the ISM and the band strength of carbon-related absorption features, especially the interstellar UV bump which is still an open point.

\section{Conclusions}

UV irradiation of hydrogenated amorphous carbon (HAC) materials produced by the laser ablation is an analog of the interaction of the cosmic carbonaceous nanoparticles with UV radiation in the diffuse interstellar space. Analytical studies by HRTEM show the structural modifications of irradiated HAC materials. The graphene layer length increases from $0.42-0.7$ to $2.2-$ $2.9 \mathrm{~nm}$ with an UV irradiation dose up to $1.0 \times 10^{23} \mathrm{eV} \mathrm{cm}^{-2}$. Graphitic fibers are observed as well. The $\mathrm{H} / \mathrm{C}$ ratio is about 0.6 for HAC materials produced at a laser power density of $2 \times 10^{8} \mathrm{~W} \mathrm{~cm}^{-2}$ and it can reach 0.82 with increasing the laser power density up to $3 \times 10^{10} \mathrm{~W} \mathrm{~cm}^{-2}$. Hydrogen content and $\mathrm{sp}^{3} / \mathrm{sp}^{2}$ ratio decrease with the strong UV irradiation. Due to the graphitization of HAC materials, UV irradiation in high vacuum corresponding to $21-33 \%$ of the average UV radiation dose in diffuse ISM produces a new band at $4.6 \mu \mathrm{m}^{-1}$ making them candidates of the carrier of the UV bump at $4.6 \mu \mathrm{m}^{-1}$ in the interstellar extinction. For reproducing the UV bump at $4.6 \mu \mathrm{m}^{-1}$ toward the diffuse ISM, the required amount of carbon based on our irradiated samples is between 108 and 269 ppm relative to hydrogen, which is higher than the carbon abundance available for interstellar dust grains.

Acknowledgements. This work was supported by the Egyptian government in cooperation with Astrophysical Institute and University Observatory, Friedrich Schiller University Jena, Germany. We thank the referee Dr. V. Mennella, for his valuable comments and suggestions.

\section{References}

Angus, J. C., \& Jansen, F. 1988, J. Vacuum Sci. Technol., 6, 1778 Buch, V. 1989, ApJ, 343, 208

Cairns, R. B., \& Samson, J. A. R. 1966, J. Opt. Soc. Am., 56, 1568 Cardelli, J. A., Clayton, G. C., \& Mathis, J. S. 1989, ApJ, 345, 245 Cardelli, J. A., Meyer, D. M., Jura, M., \& Savage, B. D. 1996, ApJ, 467, 334 Chhowalla, M., Wang, H., Sano, N., et al. 2003, Phys. Rev. Lett., 90, 155504 Chiar, J. E., Tielens, A. G. G. M., Whittet, D. C. B., et al. 2000, ApJ, 537, 749 Colangeli, L., Schwehm, G., Bussoletti, E., et al. 1990, ApJ, 348, 718

Colangeli, L., Bussoletti, E., Pestellini, C. C., et al. 1997, Adv. Space Res., 20, 1617

Dartois, E., \& Muñoz-Caro, G. M. 2007, A\&A, 476, 1235

Dartois, E., Muñoz Caro, G. M., Deboffle, D., \& d'Hendecourt, L. 2004, A\&A, 423, L33

Dartois, E., Geballe, T. R., Pino, T., et al. 2007, A\&A, 463, 635

Draine, B. T., \& Lee, H. M. 1984, ApJ, 285, 89

Duley, W. W., \& Seahra, S. 1998, ApJ, 507, 874

Ehrenfreund, P., \& Charnley, S. B. 2000, ARA\&A, 38, 427

Fitzpatrick, E. L. 1999, PASP, 111, 63

Fitzpatrick, E. L. 2004, in Astrophysics of Dust, ed. A. N. Witt, G. C. Clayton, \& B. T. Draine ASP Conf. Ser., 33, 309

Fitzpatrick, E. L., \& Massa, D. 1986, ApJ, 307, 286

Fitzpatrick, E. L., \& Massa, D. 1990, ApJS, 72, 163

Fitzpatrick, E. L., \& Massa, D. 2005, AJ, 130, 1127

Fitzpatrick, E. L., \& Massa, D. 2007, ApJ, 663, 320

Furton, D. G., Laiho, J. W., \& Witt, A. N. 1999, ApJ, 526, 752

Greenberg, J. M., \& Li, A. 1999, Adv. Space Res., 24, 497

Habing, H. J. 1968, Bull. Astron. Inst. Netherlands, 19, 421

Hecht, J. H. 1986, ApJ, 305, 817

Iida, Y., \& Yeung, E. S. 1994, Appl. Spectrosc., 48, 945

Jacob, W., \& Unger, M. 1996, Appl. Phys. Lett., 68, 475

Jäger, C., Mutschke, H., \& Henning, T. 1998, A\&A, 332, 291

Jäger, C., Henning, T., Schlögl, R., \& Spillecke, O. 1999, J. Non-Crystalline Solids, 258, 161

Jäger, C., Mutschke, H., Henning, T., \& Huisken, F. 2008, ApJ, 689, 249

Jenniskens, P. 1993, A\&A, 274, 653

Jones, A. P., Duley, W. W., \& Williams, D. A. 1990, QJRAS, 31, 567

Kim, S., \& Martin, P. G. 1996, ApJ, 462, 296

Llamas-Jansa, I., Jger, C., Mutschke, H., \& Henning, T. 2007, Carbon, 45, 1542

Mathis, J. S., Mezger, P. G., \& Panagia, N. 1983, A\&A, 128, 212

Mennella, V., Colangeli, L., Blanco, A., et al. 1995, ApJ, 444, 288

Mennella, V., Colangeli, L., Palumbo, P., et al. 1996, ApJ, 464, L191

Mennella, V., Baratta, G. A., Colangeli, L., et al. 1997, ApJ, 481, 545

Mennella, V., Colangeli, L., Bussoletti, E., Palumbo, P., \& Rotundi, A. 1998, ApJ, 507, L177

Mennella, V., Brucato, J. R., Colangeli, L., \& Palumbo, P. 1999, ApJ, 524, L71

Mennella, V., Brucato, J. R., Colangeli, L., \& Palumbo, P. 2002, ApJ, 569, 531

Mikhailov, E. F., Valsenko, S. S., \& Kiselev, A. A. 2001 (Chichester UK: John Wiley and sons, Inc.), 413

Ogmen, M., \& Duley, W. W. 1988, ApJ, 334, L117

Pendleton, Y. J., \& Allamandola, L. J. 2002, ApJS, 138, 75

Phillips, J. C. 1979, Phys. Rev. Lett., 42, 1151

Pino, T., Dartois, E., Cao, A., et al. 2008, A\&A, 490, 665

Rietmeijer, F. J. M. 1988, in Progress Toward a Cosmic Dust Collection Facility on Space Station. Report of the Workshop on Micrometeoritie Capture Experiments, held June 28-July 1, 1987, sponsored by the Lunar and Planetary Institute. LPI Technical Report 88-01 (Houston: Lunar and Planetary Institute, 3303 NASA Road 1, TX 77058), ed. I. D. R. MacKinnon, \& W. C. Carey, 53

Robertson, J. 1986, Adv. Phys., 35, 317

Robertson, J., \& O'Reilly, E. P. 1987, Phys. Rev. B, 35, 2946

Schnaiter, M., Mutschke, H., Dorschner, J., Henning, T., \& Salama, F. 1998 ApJ, 498, 486

Snow, T. P., \& Witt, A. N. 1995, Science, 270, 1455

Sofia, U. J., \& Parvathi, V. S. 2009, in ASP Conf. Ser. 414, ed. T. Henning, E. Grün, \& J. Steinacker, 236

Sofia, U. J., Wolff, M. J., Rachford, B., et al. 2005, ApJ, 625, 167

Sorrell, W. H. 1990, MNRAS, 243, 570

Valencic, L. A., Clayton, G. C., \& Gordon, K. D. 2004, ApJ, 616, 912

Wada, S., Kaito, C., Kimura, S., \& Tokunaga, A. T. 1999, Adv. Space Res., 24, 523

Whittet, D. C. B. 2004, in Bioastronomy 2002 Life Among the Stars, ed. R. Norris, \& F. Stootman, IAU Symposium, 213, 163

Zubko, V. G., Mennella, V., Colangeli, L., \& Bussoletti, E. 1996, MNRAS, 282, 1321 